\title{
Deformation of crystals: Connections with statistical physics
}

\author{
James P. Sethna, ${ }^{1}$ Matthew K. Bierbaum, ${ }^{1}$ \\ Karin A. Dahmen, ${ }^{2}$ Carl P. Goodrich, ${ }^{3}$ Julia R. \\ Greer ${ }^{4}$ Lorien X. Hayden, ${ }^{1}$ Jaron P. \\ Kent-Dobias, ${ }^{1}$ Edward D. Lee, ${ }^{1}$ Danilo B. \\ Liarte, ${ }^{1}$ Xiaoyue Ni, ${ }^{4}$ Katherine N. Quinn, ${ }^{1}$ \\ Archishman Raju, ${ }^{1}$ D. Zeb Rocklin, ${ }^{1}$ Ashivni \\ Shekhawat, ${ }^{5}$ and Stefano Zapperi ${ }^{6}$
}

Xxxx. Xxx. Xxx. Xxx. YYYY. AA:1-29

This article's doi:

$10.1146 /(($ please add article doi $))$

Copyright (C) YYYY by Annual Reviews. All rights reserved

${ }^{1}$ Laboratory of Atomic and Solid State Physics, Cornell Univ., Ithaca, NY 14853-2501, USA; email:sethna@lassp.cornell.edu

${ }^{2}$ Physics Dept., Univ. of Illinois at Urbana-Champaign, Urbana, IL, USA, 61810 ${ }^{3}$ School of Eng. and Applied Sciences, Harvard Univ., Cambridge, MA 02138

${ }^{4}$ Division of Engineering and Applied Sciences, California Institute of Technology, Pasadena, California 91125, USA

${ }^{3}$ Dept. of Physics, Univ. of Milano, Via Celoria 16, 20133 Milano, Italy

${ }^{3}$ Dept. of Physics, Univ. of Milano, Via Celoria 16, 20133 Milano, Italy

${ }^{5}$ Materials Sciences Division, Lawrence Berkeley National Laboratory, Berkeley, California 94720, USA

${ }^{6}$ Dept. of Physics and Center for Complexity and Biosystems, Univ. of Milano, Via Celoria 16, 20133 Milano, Italy

\section{Keywords}

plasticity, crystals, critical phenomena, irreversible deformation, dislocation,work hardening, renormalization group

\section{Abstract}

We give a bird's-eye view of the plastic deformation of crystals aimed at the statistical physics community, and a broad introduction into the statistical theories of forced rigid systems aimed at the plasticity community. Memory effects in magnets, spin glasses, charge density waves, and dilute colloidal suspensions are discussed in relation to the onset of plastic yielding in crystals. Dislocation avalanches and complex dislocation tangles are discussed via a brief introduction to the renormalization group and scaling. Analogies to emergent scale invariance in fracture, jamming, coarsening, and a variety of depinning transitions are explored. Dislocation dynamics in crystals challenges nonequilibrium statistical physics. Statistical physics provides both cautionary tales of subtle memory effects in nonequilibrium systems, and systematic tools designed to address complex scale-invariant behavior on multiple length and time scales. 


\section{Contents}

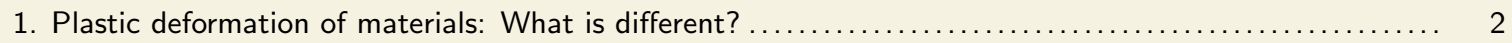

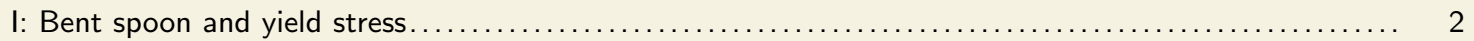

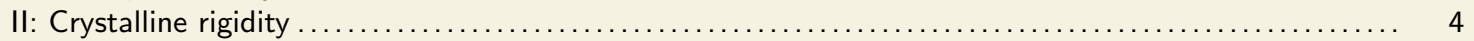

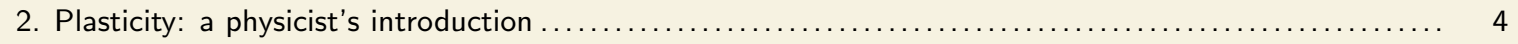

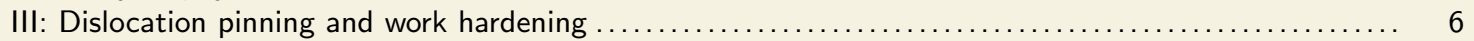

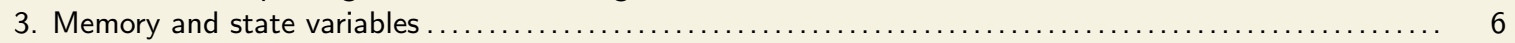

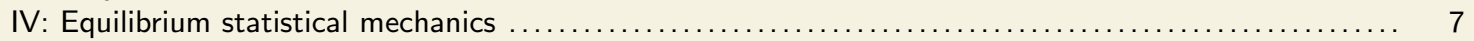

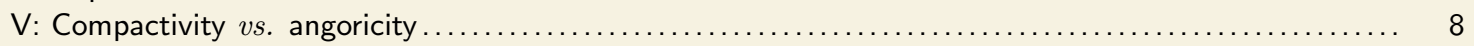

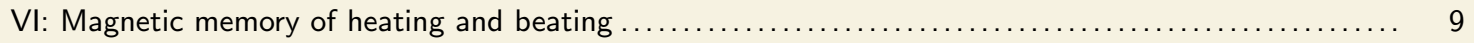

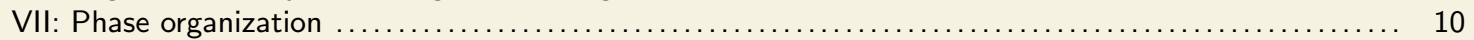

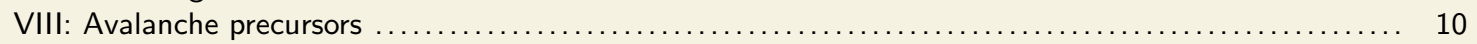

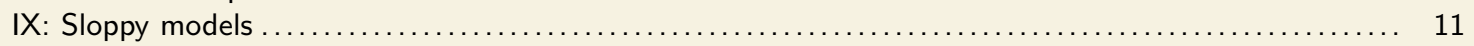

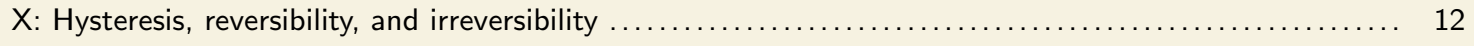

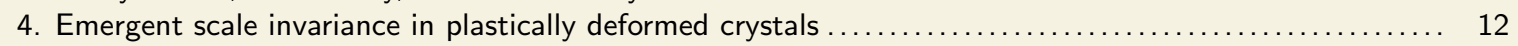

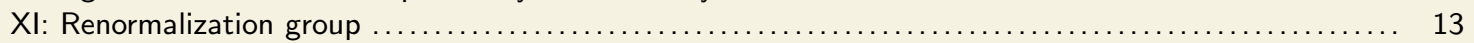

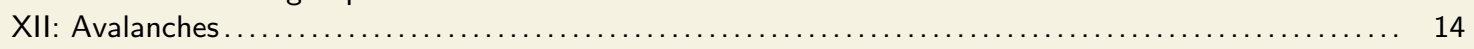

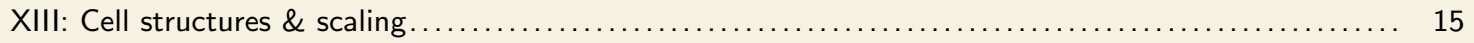

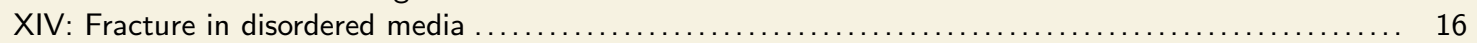

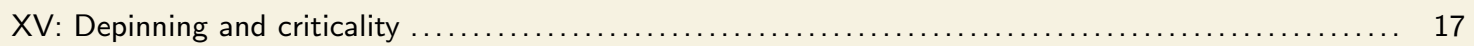

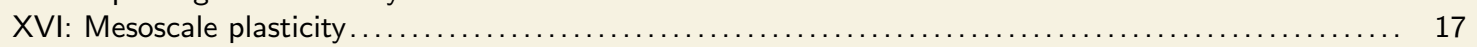

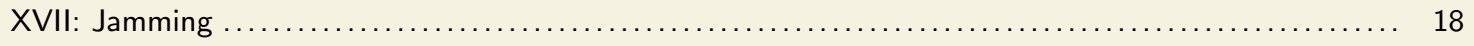

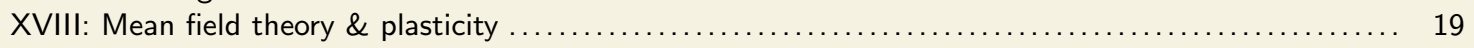

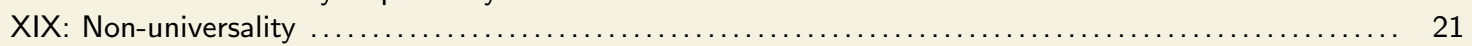

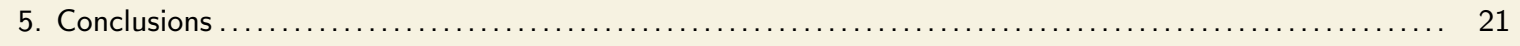

\section{Plastic deformation of materials: What is different?}

Many of us, when trying to scoop ice cream, have induced plastic deformation: the spoon remains bent (Figure I). Speculations about strategies for understanding our bent spoon represent the topic of this review.

\section{Figure I: Bent spoon and yield stress}

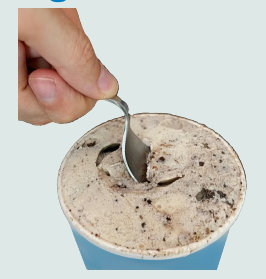

A metal spoon will spring back into its original shape under ordinary use, but in hard ice cream one may bend the spoon too far for it to recover. The spoon is made up of many crystalline grains, each of which has a regular grid of atoms. To permanently deform the spoon, atomic planes must slide past one another. This happens through the motion of dislocation lines. The dynamics, interactions, and entanglement of these dislocation lines form the microscopic underpinnings of crystal plasticity, inspiring this review.

The physics and engineering communities have historically focused on studying how plastic deformation is similar to simpler systems. Elastic materials respond to stress fields via strain fields; liquids respond via viscous strain rates; complex fluids are often describ- 
able via frequency-dependent viscoelastic responses. Many properties of crystals, magnets, liquid crystals, superconductors, superfluids, and field theories of the early universe can be described by focusing on long length scales and assuming that the materials are locally close to equilibrium. Physicists use generalized hydrodynamics $(1,2,3)$ or Landau theory (4), and have systematized how conserved quantities (particle number, momentum, energy) and broken symmetries (magnetization, crystalline order) are assembled into order parameter fields. Engineers apply continuum field theories (5) using phenomenological material models to study materials behavior. They incorporate state variables like dislocation density, yield stress, or texture to describe the behavior of real materials at macroscopic scales. These models have been effectively incorporated into the computational frameworks used to design everything from spoons to airplanes. In Section 2 we shall provide a physicist's introduction to the challenges posed by plasticity in metals, touching or ignoring many crucial features (slip systems, partial dislocations, grain boundaries, etc), but emphasizing how the broken translational symmetry of crystals make them unusual among condensed matter systems.

Inspired by plasticity and failure of practical materials, the statistical physics community has studied a remarkable variety of rigid nonequilibrium systems evolving under stress. They have focused on what makes magnets, sand, and other rigid systems under forcing different from simpler systems (6). This article summarizes results from many of these studies, from magnetic hysteresis to jamming of granular materials, in brief vignettes similar to Figure I.

There are striking regularities that emerge in the deformation and failure of materials that lure us to search for a systematic theory. First, despite a bewildering variety of material morphologies, most structural materials share certain characteristic damage thresholds. Plastic deformation in practical materials does not arise at arbitrarily small applied stresses: the onset of deformation characterizes the yield strength of materials (Figure III). The yield stress is an approximation; creep and fatigue allow for hysteretic changes below the yield stress. But by ignoring certain physical mechanisms (e.g. things like vacancy diffusion and cross slip that typically go away at low temperatures) we can study a theory that predicts a sharp threshold in quasi-static deformations, and hence provides an effective explanation of the observed, but less sharp, transitions in practical materials.

The yield stress represents the division between elastic and plastic, the division between reversible and hysteretic macroscopic deformations. Fundamentally, equilibrium systems forget their history: all other microscopic degrees of freedom are 'slaves' to the state variables. The chaotic local dynamics on the atomic scale scramble the history of how the material was prepared; only those quantities preserved by the dynamics can matter for the long-time macroscopic behavior. In contrast, plastically deformed materials are not in local equilibrium, and their properties depend on their history. The blacksmith hammering the red-hot horseshoe and quenching it into water is altering a complex microstructure which governs its toughness and strength; a cast-iron horseshoe of identical chemical composition could be brittle. In Section 3 we shall briefly and broadly review how the history of deformation has been reflected in other statistical mechanics contexts: can the memory of the material's past be effectively summarized in a finite number of continuum variables? What can we glean from these other systems about what might be special about the dislocation configurations left behind after yielding and unloading in crystals?

Statistical physicists adore power laws and emergent scale invariance; when systems appear the same on different scales, we have powerful, systematic tools to quantitatively predict the resulting behavior. In Section 4 we shall examine evidence that dislocation

Strain: Fractional amount $\epsilon$ a crystal is stretched or sheared, twisted or

compressed.

Stress: Force per unit area $\sigma$ applied to a crystal. Both $\epsilon$ and $\sigma$ are tensors, a fact we shall largely ignore.

Equilibrium: In this paper, 'equilibrium' and 'nonequilibrium' will ordinarily refer to thermal equilibrium (Figure IV); most of our systems reach mechanical equilibrium - a metastable state.

Rigid: Rigid systems have an energy cost to deforming some continuum field magnetic, elastic, charge-density distortion, superfluid, ...

Burgers vector: Topological charge of a dislocation: number of extra planes of atoms crossed when encircling the defect (7, Sect. 9.4).

Creep: Dislocation motions, like dislocation climb, that proceed slowly and remain present below the traditional yield stress. They are usually thermally activated. A component of rate-dependent plasticity. 
Figure II: Crystalline rigidity

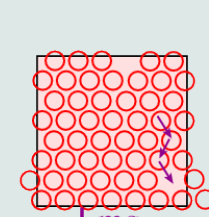

(a) $\downarrow \mathrm{mg}$

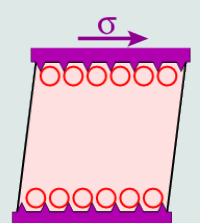

(b)

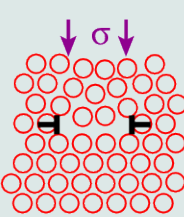

(c)

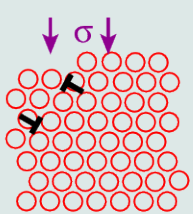

(d) (a) Under a force like gravity that couples to the mass density, an equilibrium crystal can flow like a liquid (at a rate linear in the gravitational force), via vacancy diffusion. (This is true at temperatures above the roughening transition (8), where the equilibrium surfaces

all have steps that can absorb and emit vacancies.) (b) Crystals are rigid to shears $\sigma$ that couple to the lattice (i.e., the broken translational symmetry). (c) A crystal sheared or compressed more than half a lattice constant will, in equilibrium, lose a plane of atoms. Here a dislocation loop surrounds a disk of missing atoms, which can grow only by climb, again via thermally activated vacancy diffusion. (d) The dominant shear relaxation at low temperatures is glide, where a dislocation loop can grow along a slip plane to relieve stress without involving vacancies (see Figure VIII(a)). The nucleation rate for the dislocation loops in (c) and (d) goes to zero faster than linearly as the compression goes to zero; there is no linear viscous flow response to forces that couple to the lattice. This distinguishes crystals from liquids.

Slip plane: The plane accessible to a dislocation of a given Burgers vector via glide.

Slip systems: The set of slip planes for the low-energy Burgers vectors for a given crystal symmetry. The initial deformation of pure metals usually 'activates' only one or a few slip systems. evolution and plastic flow exhibits just this kind of scale invariance - power-law distributions of dislocation avalanches and complex, perhaps scale invariant morphologies. We shall discuss plasticity models which explain this as self-organized criticality, controlled by work hardening, and also discuss the possibility that these effects are due to a proximity to a critical failure stress. We shall discuss the theoretical renormalization-group framework that has been used to analyze emergent scale invariance, introduce related non-equilibrium statistical mechanics systems that have been studied using these methods, and speculate about possible connections to plastic deformation in crystals. There we shall also discuss the tension between the universality traditionally expected at critical points and the strong non-universal dependence on material, morphology, and loading seen at the yielding transition.

\section{Plasticity: a physicist's introduction}

Almost any collection of atoms or molecules, when cooled, will form into a rigid, solid object. This rigidity is an unappreciated, profound state of matter. Much is made of the precision measurements made possible by the Josephson effect in superconductors or the quantum Hall effect in two-dimensional electron gases. But the rigidity of solids underlies almost every measurement we do. For example, it allows the mirrors of LIGO to be held multiple kilometers apart with an accuracy much smaller than an atomic nucleus. From bridges to bones, the rigidity of solids underpins our world.

Rigidity may seem straightforward. Atoms and molecules bond together, and breaking bonds demands energy - either thermal energy to melt the material, or external forces to bend, shear, or fracture it. For glasses and amorphous materials, the key question is what makes them different from liquids. Structurally similar to liquids, how and why do they get trapped into a subset of the possible atomic configurations? What is the nature of the glass transition, where the material stops flowing and develops an elastic rigidity to small

Sethna et al. 
external forces? We shall touch upon current 'jamming' theories regarding the rigidity of glasses in Figure XVII.

Even the rigidity of crystals is subtle. Each atom in a crystal knows its place: the regular array of atoms cannot flow. A crystal has no linear shear-rate response to an external strain of the crystalline lattice (Figure IIa, b). But it will shear under high loads, plastically deforming through the motion of dislocations (Figure IIc, d). What divides elastic from plastic?

How far can one strain a crystal before it will plastically deform - rearranging its lattice to lower its energy? It is easy to see, but quite surprising, that the limiting stability region of an equilibrium crystal is microscopic. Consider a crystal being compressed vertically (Figure IIc,d), with free boundary conditions along the horizontal. It is clear that the lowest free energy configuration of the crystal will change - removing one horizontal plane of atoms, and moving them to the edge - once the compressive deformation reaches a lattice constant. Similarly, a bent crystal can lower its energy by developing a low-angle grain boundary once the net deformation is of order the lattice constant times the logarithm of the number of atomic layers. This is quite different from most broken symmetry systems; magnets, superfluids, and superconductors can be twisted at their edges by large angles or phases before they prefer to generate defects to relax. Crystalline rigidity is frail. ${ }^{1}$

This frailty is not just a theoretical curiosity. Although perfect single crystals (such as nanowhiskers) can support enormous strains before yielding, high-quality nearly perfect fcc crystals have very low thresholds for plastic deformation. Consider a dislocation line segment in a crystal, pinned at two points a distance $L$ apart, perhaps by inclusions, impurities, or other dislocations. (We ignore effects due to the discreteness of the crystalline lattice.) The component of the external stress $\sigma$ that couples to the dislocation will cause it to bow out, forming a roughly circular arc, until its line tension and interaction energy balances the external stress at a rough 'curvature diameter' $D_{c}(\sigma)$. When $D_{c}<L$, the loop grows without bound, sometimes pinching off to form a growing dislocation loop.

The durable crystals familiar to us have rather large densities of pinning sites, often arising from the tangle of dislocations formed by previous plastic deformation. There is an energy barrier impeding the crossing of two (or more (13)) dislocations; ${ }^{2}$ they often instead merge into dislocation junctions, which act as pinning points. If the density of dislocation lines crossing a unit area is $\rho$, a dislocation passing through the tangle will typically have pinning intersection points separated by $L \sim 1 / \sqrt{\rho}$ (Figure III(a)). This implies a yield stress $\sigma_{Y} \propto \sqrt{\rho}$ - the Taylor relation (14). ${ }^{3}$ This power-law scaling relation, while simply derived, foreshadows the types of predictions derived from emergent scale invariance in

\section{Section 4 .}

As plastic deformation proceeds, the dislocation lines stretch and multiply, increasing their density and further increasing the yield stress. This work hardening makes plastic

\footnotetext{
${ }^{1}$ Smectic liquid crystals - made of stacked two-dimensional liquid layers - are also frail (9).

${ }^{2}$ There is no topological reason to prevent dislocations from crossing (7, Chapter 9), although often a jog will be left behind, which then can impede glide.

${ }^{3}$ One must note that grain boundaries can also act as pinning sites for dislocations, but the yield stress for grains of size $L$ does not scale as $1 / L$, but approximately as $1 / \sqrt{L}$ (the Hall-Petch relation), due to the need for several dislocations to cooperate in punching the lead dislocation through the wall (e.g., in a 'dislocation pileup'). This different scaling will be a challenge to scaling theories like those discussed in Section 4.
}

Peierls barrier: The barrier per unit length to dislocation glide due to the discreteness of the crystalline lattice; small for fcc metals.

Kink: The 'soliton' mediating dislocation glide across the Peierls barrier to the next lattice position. The barrier to kink motion is often tiny (10).

Jog: The soliton mediating dislocation climb, left behind, e.g., when dislocations cross. Pins the dislocation.

Stacking fault: For simple fcc metals, a deviation from the fcc ABCABC stacking - typically low energy, since the nearest-neighbor structures are unchanged.

Partial dislocations: The edge of a stacking fault, often forming the core of a dislocation, described by fractions of a Burger's vector. 
Figure III: Dislocation pinning and work hardening
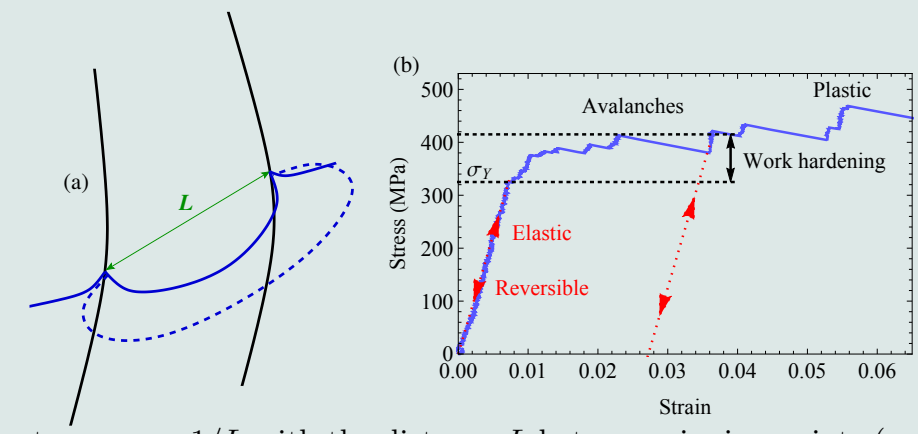

(a) Dislocations get pinned on other dislocations, dirt, inclusions, or their own jogs. Under stress, they will bow out reversibly, with a 'curvature diameter' which shrinks roughly linearly with increasing stress, until it becomes less than the distance $L$ between pins. At that point the dislocation balloons out and triggers an avalanche. This argument explains the observed scaling of the yield stress $\sigma_{Y} \sim 1 / L$ with the distance $L$ between pinning points (see e.g. Ref. (11, Chapter 10)). (b) Micropillar deformation, showing avalanches (12). The response of a material is reversible below a yield stress $\sigma_{Y}$, after which dislocation avalanches lead to plastic deformation. When unloaded, the material will respond reversibly until reloaded to roughly the previous maximum stress. Subsequent plastic flow increases the length and density of dislocations, leading to more pinning and work hardening.

Screw dislocation: A dislocation directed parallel to its Burgers vector, forming a 'spiral stair'. The screw portion of a dislocation loop can glide in any direction.

Cross slip: The motion of the screw portion of a dislocation loop into a different glide plane, often involving thermally activated restructuring of partial dislocations. deformation self-limiting in crystals; a weak spot becomes stronger when it yields. ${ }^{4}$ Hence a pristine copper wire (or an ordinary metal clothes hanger) can be bent quite easily into a tight curve. But once bent, it becomes far more resistant to further deformation; bending takes far less force than unbending.

Plastic deformation is the study of non-equilibrium collective dynamics of spatially extended topological defect lines, with long-range elastic interactions and complex constraints on the defect motions. Each of these features has separately become a focus of statistical physics in the past decades. Indeed, many of us have been inspired to study memory effects and critical phenomena in these other systems because of seeming relations to the challenging practical problems illustrated by bent spoons. Let us now proceed with a distillation of some of the key ideas developed in statistical physics that could be useful or inspirational for the study of plastic deformation of crystals.

\section{Memory and state variables}

It is a truism in metallurgy that the mechanical properties of crystals depend on their thermal and deformation history - the 'heating and beating' suffered by the material in the past (17). Mechanical plastic deformation of a crystal typically increases the threshold for further deformation (work hardening); heating it to high temperatures anneals the crystal back to a plastically soft state. As discussed in Section 2, different theories of plasticity attempt to encapsulate this history dependence into a variety of state variables. The simple sample yield stress we have discussed, for example, could be promoted into a spatially-dependent yield stress of an inhomogeneously processed specimen, or to an entire

\footnotetext{
${ }^{4}$ Metallic glasses, on the other hand, become weaker as they shear, leading to failure via slip bands $(15,16)$.
}

6 Sethna et al. 
yield surface in the six-dimensional space of stresses, or to a scalar, tensor, or slip-system specific dislocation density, etc.

The history-dependence of other rigid systems has been an active field in statistical mechanics. Some have focused on how non-equilibrium systems might be similar to equilibrium phases, with a thermodynamics resulting from a guiding principle similar to maximizing entropy. Some have explored the weird ways that particular rigid systems respond to external forcing. And some have focused on how non-equilibrium systems may be governed by critical points (Section 4), with scaling behavior similar to continuous equilibrium phase transitions.

Figure IV: Equilibrium statistical mechanics

Equilibrium systems are simple (7, Chapter 4) first because their dynamics is chaotic. Chaotic systems rapidly lose all information about their previous state, except for conserved quantities (energy, volume, number of particles, ...), and fields associated to broken symmetries (magnetization, crystalline strain, superfluid phase, ... ). Their time-average behavior then becomes a weighted average over their 'attractor'. Thermal equilibrium systems are simple second because their energy-conserving Hamiltonian dynamics preserves volume in phase space (Liouville's theorem), telling us that the attractor includes all possible states consistent with the preserved information, weighted by phase space volume (a 'maximum entropy' state). This leads directly to free energies and thermodynamics. Temperature, pressure, chemical potential, and stress arise as Lagrange multipliers to constrain the conserved energy, volume, number, and strain.

The plasticity of crystals forms the prototypical example of a nonequilibrium, historydependent rigid system. It has inspired and guided careful work on memory effects in other statistical mechanical contexts. We may hope that some of the unusual memory ramifications observed in these simpler systems might be relevant for plasticity. In this section, we briefly highlight studies of the first two types (nonequilibrium thermodynamics, vs. weird memory effects), and discuss how they might give insight into practical plasticity problems. We will turn to consider nonequilibrium critical points in Section 4.

Sam Edwards (19) proposed the most influential recent analogy between nonequilibrium systems and equilibrium statistical mechanics (Figure IV): a thermodynamic theory of packed granular powders. ${ }^{5}$ He posited a 'phase space' of force-balanced jammed arrangements of grains producing a kind of granular entropy, with volume replacing energy as the conserved quantity and temperature replaced by a compactivity field. Recent experiments $(21,18$, see Figure V) show very generally that such a description does not satisfy the zeroth law (two kinds of particles in equilibrium with a third must be in equilibrium with one another). Indeed, it is hard to see how the admittedly complicated dynamics of grain motion as it is tamped or sheared would rearrange particles to transmit extra volume effectively through the system. However, the forces between touching particles can rearrange dramatically under changing loads even for fixed particle contacts. (Puckett and Daniels (25) mention that forces and torques balance at each contact, while volume is

\footnotetext{
${ }^{5}$ Note that low-density shaken granular materials fluidize; such systems do explore their available states and in many regimes can be approximated well by theories drawing from thermodynamics $(24)$.
} 
Figure V: Compactivity vs. angoricity

\begin{tabular}{|l|c|c|c|}
\hline & Boltzmann & Edwards & HC-EB \\
\hline \hline Conserved quantity & Energy, $E$ & Volume, $V$ & Force-moment, $\hat{\Sigma}$ \\
\hline Entropy & $S=k_{B} \ln \Omega_{B}(E)$ & $S=\lambda \ln \Omega(V)$ & $S=\lambda \ln \Omega(\hat{\Sigma})$ \\
\hline Intensive quantity & Temperature & Compactivity & Angoricity \\
\hline$\frac{1}{T}=\frac{\partial S(E)}{\partial E}$ & $\frac{1}{X}=\frac{\partial S(V)}{\partial V}$ & $\alpha_{\mu \lambda}=\frac{\partial S\left(\Sigma_{\mu \lambda}\right)}{\partial \Sigma_{\mu \lambda}}$ \\
\hline Distribution & $\exp \left[-E /\left(k_{B} T\right)\right]$ & $\exp (-V / X)$ & $\exp \left(-\alpha_{\mu \lambda} \cdot \Sigma_{\mu \lambda}\right)$ \\
\hline
\end{tabular}

There has been an ambitious attempt to use statistical mechanics to derive a thermodynamics of dry grains and dense non-Brownian suspensions (18). Grains of sand do not move unless pushed; energy and temperature are unimportant. Edwards and Oakeshott (19) proposed a 'microcanonical' ensemble for powders that maximized an entropy with volume as a conserved state variable, with the distinction from phase space that the states $\Omega(V)$ were restricted to jammed configurations (see Figure XVII) (20). Recent experiments suggest that the zeroth law of thermodynamics does not hold for the resulting 'compactivity' temperature. By incorporating force and torque balances, Henkes \& Chakraborty (21) and Edwards \& Blumenfeld (22, 23) derive a thermodynamics where a force-moment tensor $\hat{\Sigma}$ plays the role of energy, and the intensive quantity $\hat{\alpha}$, named angoricity ('stress' in Greek), plays the role of temperature. The zeroth law so far appears to hold for angoricity.

Rate-independent plasticity: Deformations that happen slowly compared to dislocation glide process, but fast compared to creep; the focus of this discussion.

merely conserved globally.) This perhaps could form the basis of a thermodynamic theory (Figure V).

There are serious challenges to similar analogies between dislocations and equilibrium statistical mechanics. Dislocation energies are strongly coupled to their atomic environments; they lose heat as they nucleate, move, and tangle, and hence it would seem that no direct 'dislocation temperature' should exist (26) distinct from the thermodynamic temperature. Many of these same concerns, however, apply to granular systems $(27,28)$, glassy simulations (29) and foams (30), all of which have shown evidence of effective temperatures and maximization of entropy (20). Dislocations also exchange force with the crystal lattice; (pinned to inclusions, jogs, 'sessile' dislocation junctions, ....), presumably also arguing against a 'dislocation angoricity' (Figure V).

More prevalent in nonequilibrium statistical mechanics are studies of how particular rigid systems behave weirdly under forcing. ${ }^{6}$ These other systems have attracted interest not because of the behavior as they are unloaded and reloaded, but rather because of the character of the microstates selected by the cycle of unloading and reloading. What can we glean from these about crystal plasticity?

The textbook picture of yield stress in crystals $(11,17)$ is particularly simple. Raising the stress to the yield stress and back to zero supposedly leaves the system in precisely the same state (ignoring creep); raising the stress beyond the yield stress leads to rearrangements of dislocations into new metastable configurations. Dislocations move and tangle under

\footnotetext{
ior.

${ }^{6}$ A quantitative 'dislocation thermodynamics' would seem incompatible with such weird behav-
} 
Figure VI: Magnetic memory of heating and beating
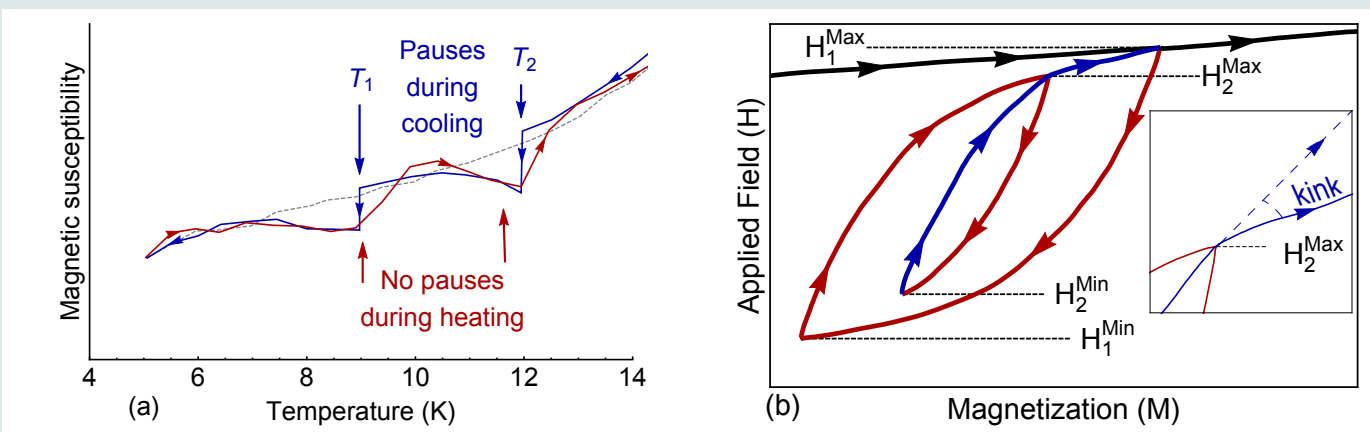

(a) Spin glasses are frustrated magnetic systems, which have modes that are active in narrow ranges of temperatures. Annealing on cooling at temperatures $T_{1}, T_{2}$ leaves fewer susceptible modes upon heating through the same temperatures (31). (b) Disordered ferromagnets at zero temperature have hysteresis loops that return to precisely the same microstates after the external field is decreased from $H^{\max _{1}}$ and returned to the previous maximum $H^{\text {max }}$, even though the avalanches differ on raising and lowering the field. This is a return-point memory of the previous external field history (32). Each return to a previous maximum results in a kink in the $M(H)$ curve.

increasing stress, like snow gets pushed when a snow plow moves forward on the street. Under decreasing forcing, the dislocation tangles presumably remain in place just as does the snow.

Magnets and other hysteretic systems have hysteresis upon decreasing and increasing the external field, but certain magnetic systems do return to precisely the same state upon reloading (the return-point memory effect, Figure $\mathrm{VIb}$ ). The avalanches they undergo upon unloading differ from those upon reloading. Upon increasing the external field $H, M(H)$ will have a kink at the point when it 'rejoins' the old trajectory. For a system trained into multiple hysteresis subloops, a kink arises upon raising the field above each (monotonically increasing) historical peak in the field history. In principle, a magnet could encode an indefinite number of such kinks, suggesting that no finite number of state variables can perfectly encode the behavior. Memory effects also arise from the thermal history in spin glasses, which encode pauses in the thermal history upon cooling (Figure VIa).

Another rigid state of matter is the charge density wave - a modulation in the electron density of a crystal whose wavelength can be unrelated (incommensurate) to the crystal lattice, and which is often pinned by impurities. The charge density wave in some materials can slide under a large enough external voltage. When slid repeatedly for a fixed pulse time $t$, they settle into a stable limit cycle. Unlike the simple model of plasticity and the return-point memory magnets, here the hysteretic behavior must be trained by a few cycles. These systems exhibit a striking collective effect, termed phase organization $(34,35,33)$; the current at the end of the training pulse always is increasing - reflecting the marginal stability of the 'first' periodic limit cycle found by local regions in the material. In the rough 
Figure VII: Phase organization

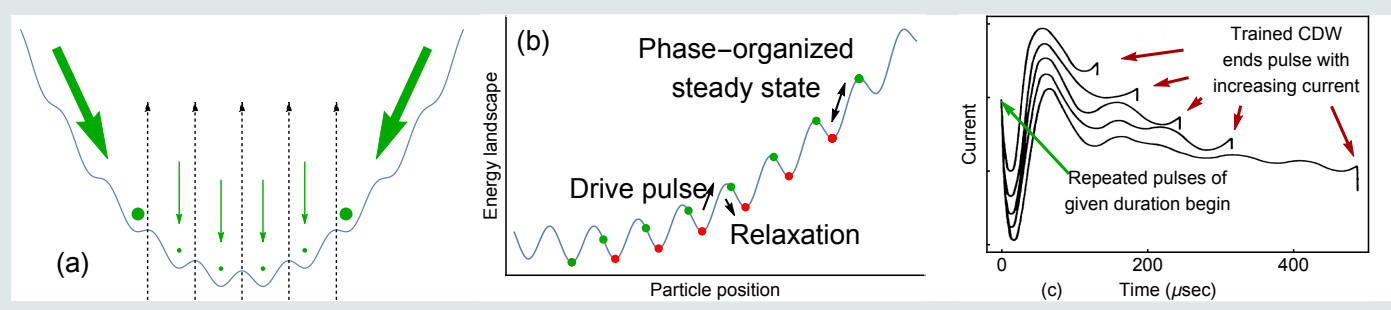

Does a nonequilibrium system dynamically dumped into a stable state select typical or unusual metastable states? (a) Consider a particle in a periodic potential, connected by a spring to a 'nail' at the origin (33). A typical initial configuration will slide down the potential until the first local minimum, which will usually be on the edge of the range of local minima - just barely stable to inward forces. A collection of such nails (representing many local regions of a material), upon random initialization, will typically have most nails on their edges - a marginally stable hyper-corner of the hyper-cube of possible system configurations. (b) For an ensemble of nailed particles trained by a pulse of a given duration (34), the pulses drive the particles up the spring potential, until a phase-organized 'edge' state in a marginally stable configuration at the end of the pulse. (c) In charge-density-wave materials, this leads to a current that always rises at the end of the pulse $(34,35)$.

hypercube of possible periodic states, the ones selected are at hypercorners (Figure VII). This example warns us that the 'stuck' states actually occupied in non-equilibrium systems can be distinct from typical metastable states in striking ways.

Figure VIII: Avalanche precursors
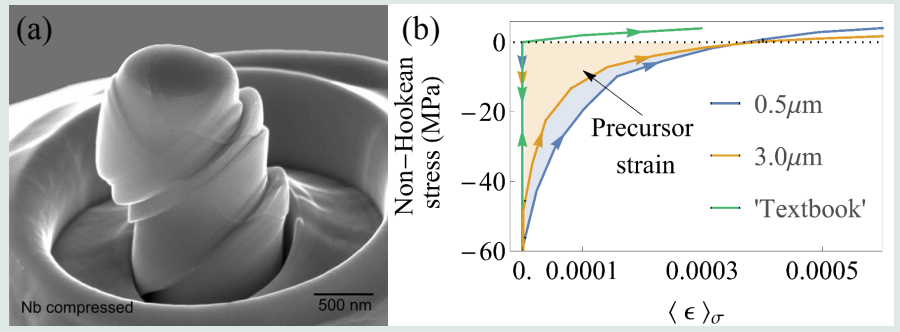

(a) Micropillars under compression (when oriented properly) yield along a single glide plane. (b) Dislocation avalanches become visible in these small-scale crystals. The macroscopic theory in textbooks (36, Ch. 26) predicts that under deformation the yield stress 'self-organizes' to the current stress $\sigma_{\max }$, and the material obeys Hooke's law as $\sigma_{i j}=C_{i j k l} \epsilon_{k l}$ upon unloading and reloading up to $\sigma_{\max }$. Here we show preliminary precision measurements by $\mathrm{Ni}$ and Greer (12) of the stress versus average strain upon reloading, for two different copper micropillars. The individual experiments clearly show precursor avalanches upon reloading, which are not a part of the macroscopic theory $(37,38,39)$. These precursors average together into the stress-strain curves shown. The smaller net precursor strain occurs in the larger pillar; it is possible that the precursor avalanches entirely disappear in macroscopic samples. 
We should note that the simple model of a yield stress, separating purely elastic behavior from irreversible deformation, and determined purely from the previous stress maximum, perhaps is oversimplified; indeed, small amounts of hysteresis and training are probably to be expected in general. For example, if the stress swings negative, the dislocations will start to rearrange at absolute stresses somewhat lower than the yield stress (the Bauschinger effect (17), Ch. 6) - reverse avalanches certainly happen as the stress direction is reversed. Since there are local stresses in a tangle due to the dislocation interactions that are comparable to the yield stress, it should be true that there are occasional 'backward' avalanches on unloading: the local material does not know when the point of zero external stress is crossed. Similarly there likely will be some precursor avalanches upon reloading below the yield stress. 'Trained' reversible states may have avalanches that differ upon loading and unloading but whose effects cancel. Figure VIII discusses preliminary results of micropillar compression experiments by $\mathrm{Ni}$ and Greer, with no avalanches upon unloading, but precursor avalanches upon reloading before reaching the previous stress maximum. (Note that these precursor avalanches could be a finite-size effect, with smaller plastic strain for larger pillars.)

Figure IX: Sloppy models
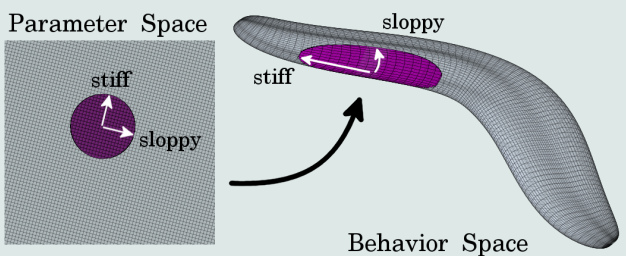

(a)

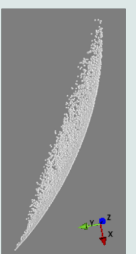

(b)

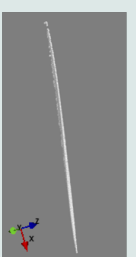

(c)
Science is possible because of a kind of parameter compression (40). The behavior of complicated systems (cell biochemistry, interatomic potentials, insect flight, critical phenomena) can be expressed in a relatively simple way, controlled by a few stiff combinations

of the microscopic parameters. (a) Information geometry describes this in terms of the manifold of all possible macroscale behaviors, which often forms a hyperribbon (41); 'sloppy' parameter combinations move along the thin directions of this model manifold. (b,c) The model manifold for fitting curves to radioactive decay forms just such a hyperribbon (Fig. from (41)). Hence, it is challenging to extract lifetimes from a sum of exponential decays. Similar decay rates and amplitudes can be 'traded' for one another along sloppy directions, with only stiff combinations constrained to keep the sum fixed. If we view the temperature and strain history in crystal plasticity as parameters, and the macroscale anisotropic strength and toughness as behavior, this parameter compression could explain the emergence of effective plasticity theories with relatively few state variables encapsulating the 'stiff' microscale combinations.

What do these examples suggest about state variables in theories of plastic flow in materials? It seems likely that crystalline dislocation networks do store their history in a way similar to that of spin glasses, magnets, and sliding charge-density waves (Figure VI). One might naively predict that deforming a crystal in some pattern as it is cooled could, upon reheating, lead to a reversed 'echo' deformation. In fact, bent crystals do not return to their original shapes when they are thermally annealed: a squashed nanopillar (Figure VIIIa) does not stand up again upon heating. Instead, the deformation history after thermal annealing is reflected in surface steps (Figure VIIIa) and (for polycrystals) in the grain orientation distribution (called texture $(42,43)$ ). We must note, though, that Shape-memory alloys 
do regain their original shapes upon reheating, due to the existence of a unique austenitic high-temperature state (44).

The fact that many historical parameters can be read from the current state does not preclude a useful low-dimensional description. Just because the current state of a material can encode its whole history does not mean details of the history affect the behavior in significant ways. For example, while a magnet after 'ring-down' (Figure VI) will show a cusp in the magnetization curve at every historical ring-down maximum, the cusps rapidly become tiny; the details of subloops of subloops of hysteresis loops are not crucial for understanding magnets. Indeed, this is a broad feature of nonlinear systems and models depending on many parameters; their behavior is usually well described by a few 'stiff' combinations of parameters (see Figure IX). Perhaps yield stress, damage, porosity and other local variables are capturing the stiff microscopic combinations governing macroscale behavior.

Figure $\mathrm{X}$ : Hysteresis, reversibility, and irreversibility

Forced viscous fluids (at low Reynold's numbers) reverse their motion when the forcing is reversed. When tiny colloidal particles are added, their trajectories reverse if they do not collide during the forward motion. Repeated oscillations at fixed amplitude can train the particles not to collide, mimicking crystals forced below their yield

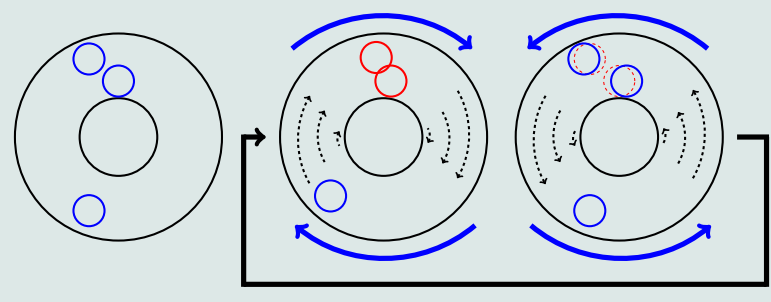
stress $(45,46,47,48,49,50,51)$. Increasing the amplitude past the previous maximum training point yields new colloidal collisions. Similar transitions (except with hysteresis in the trained state) have been seen in amorphous solids $(52,53,54,55,56,57)$, granular systems $(58,59,60,61)$, dislocations (62), and super-conducting vortices $(63,64,65,66,67,68,69)$. If the density of colloidal particles is high, or the shear amplitude is large, each collision can trigger on average one or more collisions in the next oscillation, leading to a Reversible-to-Irreversible Transition (RIT), also observed in the other systems listed above; the necessary cycling time, and the length scales of the correlated rearrangements, both diverge with power laws as discussed in Section 4.

Finally, we must mention recent work on Reversible-to-Irreversible Transitions (RITs, Figure X). ${ }^{7}$ These are systems that can be 'trained' into periodicity under low amplitude cycles. In many systems, the number of cycles to train the system can grow to infinity at a critical amplitude, after which irreversible changes continue forever. This is our first example of a critical point exhibiting power laws and scaling functions - the topic of Section 4 .

\section{Emergent scale invariance in plastically deformed crystals}

\footnotetext{
${ }^{7}$ Here we must distinguish 'local irreversibility' from rearrangements like avalanches which do not reverse along the same path upon unloading, and 'global irreversibility' when the loading gets large enough that cycling never settles down. The RIT in most cases is a transition from the former to the latter. 
Figure XI: Renormalization group

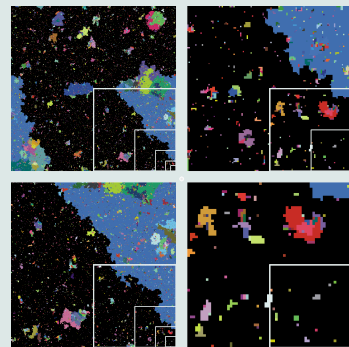

(a)

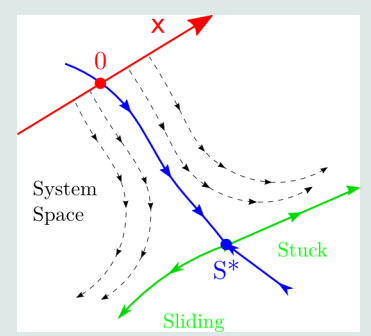

(b)
The renormalization group (RG) (70) is an amazing abstraction which works in a 'system space', with dimensions parameterizing both different possible statistical models and real experiments. The RG studies how the rules governing a system change with length scale. By coarse-graining (ignoring microscopic degrees of freedom up to a scale $b$ ), one derives rules with renormalized parameters - a dynamical flow in system space (arrows in b). (a) A RG fixed point $S^{*}$ exhibits scale invariance, so for example the avalanches in space shows a statistical self-similarity upon zooming in to the lower right-hand corner. (71)

(b) Tuning a parameter moves along a line (red) in system space; if it crosses the 'stable manifold' of $S^{*}$ (green) the system will be self-similar after coarse-graining to long length scales.

Consider some observable $Z$ (say the avalanche size distribution) as a function of parameters $x$, and $y$, (which could be avalanche size, system size, stress, or the work-hardening coefficient). If $Z, x$ and $y$ are eigenvectors of the linearized flow around $S^{*}$, then under coarse-graining $Z(x, y)=b^{-\lambda_{z}} Z\left(x b^{\lambda_{x}}, y b^{\lambda_{y}}\right)$, where $\lambda$ is the corresponding eigenvalue. If we coarse grain until $x$ flows to one (so $x b^{\lambda_{x}}=1$ ), then

$$
Z=x^{\frac{\lambda z}{\lambda x}} Z\left(1, y x^{-\frac{\lambda y}{\lambda x}}\right)=x^{\frac{\lambda z}{\lambda x}} \mathcal{Z}\left(y x^{-\frac{\lambda y}{\lambda x}}\right), \text { or alternatively } Z=y^{\frac{\lambda z}{\lambda y}} \tilde{\mathcal{Z}}\left(y x^{-\frac{\lambda y}{\lambda_{x}}}\right)
$$

The observable is a power law times a universal scaling function $\tilde{\mathcal{Z}}$ of an invariant combination of parameters $y x^{-\lambda_{y} / \lambda_{x}}$. In plasticity, the probability $P \equiv Z$ of an avalanche of size $S \equiv y$ with a strain hardening coefficient $\Theta \equiv x$ would be $P(S \mid \Theta)=S^{-\tau} \mathcal{P}\left(S / \Theta^{-d_{f} \nu}\right)$, with $\mathcal{P} \equiv \tilde{\mathcal{Z}}, \tau \equiv-\lambda_{z} / \lambda_{y}$, and $\mathrm{d}_{f} \nu \equiv-\lambda_{y} / \lambda_{x}$.

When the external stress is raised above the yield stress, clear signs of collective behavior arise. Dislocations stretch, rearrange, and entangle to mediate plastic deformation, and their entanglement at least at first raises the yield stress (work hardening, Figure III). Furthermore, there are two indications that the plastic deformation is associated with an emergent scale invariance (Figure XI), with self-similar behavior on a broad range of length and time scales.

First, as in many rigid non-equilibrium systems, crystals respond to external stress via dislocation avalanches (Figure XII). These avalanches have been observed in bulk ice crystal plasticity (80) and micropillar plasticity of a variety of fcc and bcc metals (81, 73). They come in a significant range of sizes, with a power-law probability distribution of the net slip $P(S) \sim S^{-\tau}$. The avalanches have a spatial fractal dimension $d_{f}$ less than three $(72,82)$, mostly extending along slip bands. Such avalanches are seen in many other rigid statistical mechanical models under forcing (Figure XII).

This power-law distribution of slip sizes does not extend forever. Were the distribution a pure power law, the fraction of slip produced by avalanches larger than a size $S_{0}$ would be $\left.\int_{S_{0}}^{\infty} S P(S \mid L) \mathrm{d} S \propto S^{2-\tau}\right|_{S_{0}} ^{\infty}$; since $1<\tau<2$ this would suggest that plastic deformation should be dominated by the largest events. The fact that the fractal dimension $d_{f}$ of 
Figure XII: Avalanches

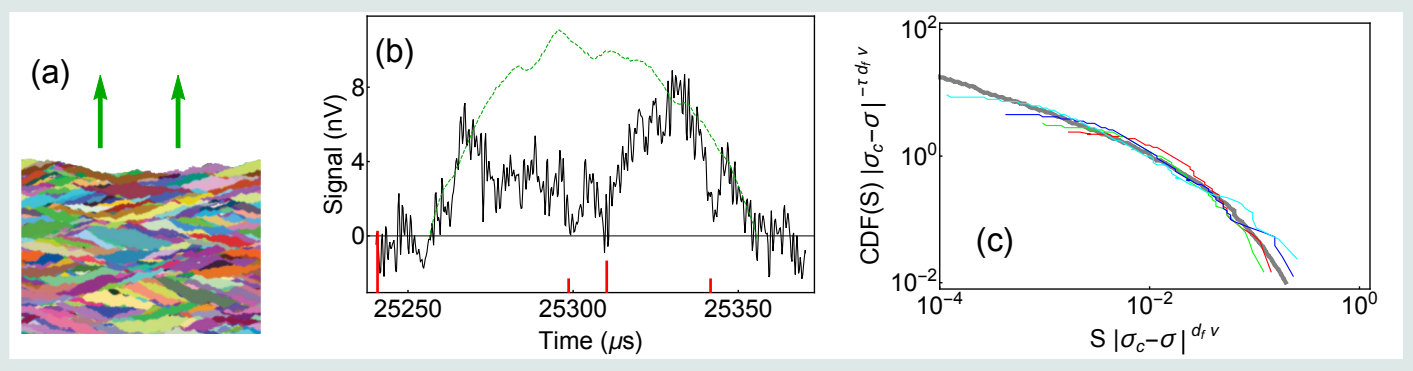

We call transitions from one metastable state to another under forcing avalanches if they span a broad range of sizes. They arise not only in plastic slip events $(72,73)$, but also earthquakes $(74,75)$, fracture $(76,77)$ and many other systems (71). (a) Avalanches in a front depinning transition (78) (e.g., coffee invading a napkin dipped into the cup). (b) A magnetic avalanche (79), illustrating the fractal structure in time $t$. The avalanche appears as a self-similar sequence of smaller events, each barely triggering the next (some large trigger events marked by red bars): here a conjunction of two medium-sized avalanches, which are in turn conjunctions of small avalanches, etc. The green curve is the signal $\langle V(t)\rangle$ averaged over all avalanches of the same duration $T$; scaling (71) predicts $\langle V(t \mid T)\rangle \sim T^{1-d_{f} / z} \mathcal{V}(t / T)$, where $z$ is a dynamic critical exponent. (c) Scaling plot for dislocation avalanche sizes, from micropillar experiments (73). The avalanche size cutoff grew with increasing stress (different colors), quantitatively following the mean-field prediction $P(S \mid \sigma)=S^{-\tau} \mathcal{P}\left(S /\left(\sigma_{c}-\sigma\right)^{-d_{f} \nu}\right)$ (grey curve). Hence, avalanches can display scale-invariance in time, space, and size.

the avalanches is below three provides one natural cutoff. For a sample of size $L$, or a sample with avalanche-blocking internal structures, such as grain boundaries, of size $L$, the biggest avalanches will be of size $L^{d_{f}}$. Think of a simple model for micropillar deformation (Figure VIII), where an avalanche induces one layer to slip over another over an area $S$ along a slip direction. The largest avalanche will span the pillar, so $S \sim L^{2}$ - but the pillar has changed height by only a few Ångstroms. More specifically (see Figure XI) the scaling distribution of avalanche sizes, cut off by a length $L$ (system size, grain size, ...), should take the form $P(S) \sim S^{-\tau} \mathcal{P}\left(S / L^{d_{f}}\right)$. In many cases, it is believed (72) that the dominant cutoff in many cases is due not to the system size, but to work hardening. As an avalanche proceeds, it increases the dislocation density in its immediate environment, raising the stress needed to produce further deformation. A work-hardening cutoff is also described by a scaling form $P(S) \sim S^{-\tau} \mathcal{P}\left(S / \Theta^{-d_{f} \nu}\right)$, where $\Theta=\mathrm{d} \sigma_{Y} / \mathrm{d} \epsilon$ is the strain-hardening coefficient (the slope of the stress-strain curve due to work hardening).

The second indication of emergent scale invariance is the complexity of the dislocation tangles that emerge under plastic deformation. They are not homogeneous tangles of spaghetti - they develop correlated patterns with structure on many length scales. Figure XIII discusses a characteristic cell structure morphology found in deformed fcc metals. It is not yet clear whether cell structures are themselves self-similar scale invariant fractals $(84,87,86)$ or whether they are similar to rescaled versions of the same system at 
Figure XIII: Cell structures \& scaling

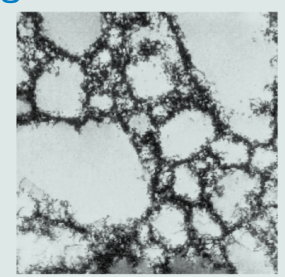

(a)

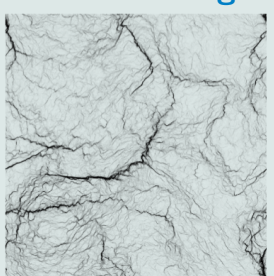

(b)
When dislocations interact they don't just form a giant ball of spaghetti (smoothly varying distributions of highly entangled defects) but instead form interesting cell structures, with dislocation-poor cell interiors surrounded by dislocation-rich walls. Since these structures contain most of the defect content of the material they dominate plastic deformation under load, with the walls providing a rigid

backbone to the softer cell interiors (83). (a) Just as the avalanches of dislocations (see Figure XII) occur on all time-scales, these cell structures display structures on many length-scales [TEM micrograph of deformed copper, from (84)]. Two different methods have been used to analyze cell structures. When analyzed using a single length scale, universal distributions emerge that are independent of material, loading process, or strain (85), refining with strain instead of coarsening with time (Figure XIX). An analysis using box-counting of the dislocation density, others find that these cell structures exhibit fractal morphology (84).

(b) Continuum models, such as that by our group (86), are able to qualitatively reproduce the complex fractal cell morphologies of real materials using grossly simplified models of dislocation dynamics. Our dislocation densities, misorientations, and other physical quantities can be described using power-laws and scaling functions. We also reproduce the universal distributions and refinement observed by single-length scaling experimental analyses.

different strains (85) (like coarsening, see Figure XIX), the complex and yet patterned form of the tangles clearly indicates the need for a theory that embraces structures on different scales.

Systems with an emergent scale invariance - that 'look the same' at different length scales and time scales - are a central focus of statistical physics. Figure XI briefly summarizes key ideas distilled from an enormous, sophisticated literature on a wide variety of systems. At a continuous transition between two qualitative behaviors (e.g. stuck to sliding as stress is tuned), many systems will exhibit scale-invariant fluctuations with regions small and large alternating between the two qualitative behaviors. Renormalization group methods use this emergent self-similarity to predict power-law behavior of functions with one control parameter (like $P(S) \sim S^{-\tau}$ above) and predict scaling forms for properties depending on more than one parameter (like $P(S \mid L) \sim S^{-\tau} \mathcal{P}\left(S / L^{d_{f}}\right)$ ).

Our first example is drawn from another materials challenge, using scaling ideas to explain various aspects of fracture. First, much attention has been spent on fractal analyses of the resulting fracture surfaces $(90,91,92,93,94,95,96,97,98)$. Two rival theories of crack growth in disordered materials materials $(99,100,101,102)$ each turned out to describe the fractal height fluctuations observed in experiments (93, 94, 97, 103, 104, 105, 106, 107, 108, 109), one at short distances and one at longer distances. A crossover scaling theory $(110,111)$ produces a unified description of experimental height correlations on intermediate length scales. Second, in brittle materials, crack nucleation is studied with extreme value statistics $(112,113,114)$ - the distribution of failure strengths is described by universal Gumbel and Weibull distributions, which recently have been viewed as renormalization-group fixed points $(115,116)$.
Process zone: The damaged zone near the tip of a crack, where linear elastic theory breaks down. For concrete, this can be nearly a meter in size. 
Figure XIV: Fracture in disordered media

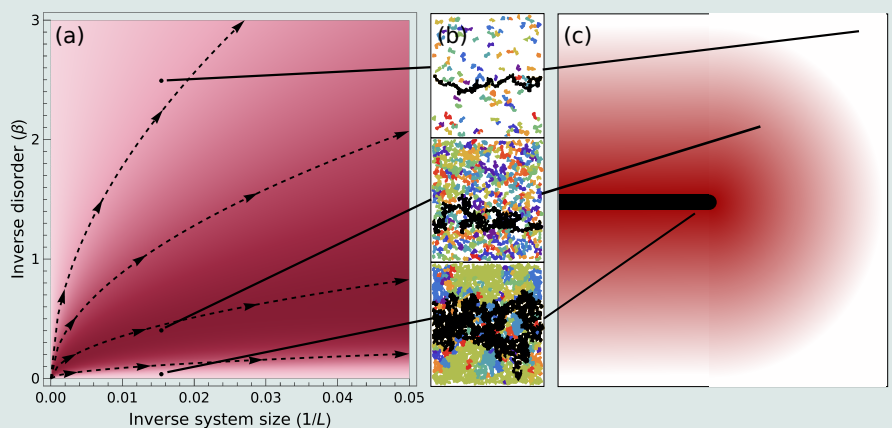

Quasi-brittle materials like concrete are disordered, with a broad distribution of microscopic strengths (88) parameterized by inverse disorder $\beta$. Low disorder or large sample size correspond with brittle, nucleated fracture [(a) upper left, (b) top], while high disorder or small size correspond with uncorrelated percolationlike fracture [(a) lower boundary, (b) bottom]. The crossover between these regimes shows large avalanches [(b) middle], whose mean second moment is indicated by red intensity. The RG flows [(a) arrows] predict that the distribution of avalanche sizes $P(s \mid \beta, L)$ has a crossover scaling form $P(s \mid \beta, L)=s^{-\tau} \mathcal{P}\left(\beta L^{1 / \nu_{f}}, s L^{-1 / \sigma \nu_{f}}\right)$; no phase boundary separates the two regimes. (c) This scenario may also apply to the damage (schematically indicated by red intensity) in the region around a growing crack for an infinite system. Near the growing crack tip, stress is high and the material is reduced to rubble (percolation), while far from the crack tip the stress is small and the material undamaged. The same RG crossover scaling analysis should allow us to develop a quantitative scaling theory of this damaged process zone (89).

The third scaling approach to fracture describes quasi-brittle materials like concrete, in which the strength of local regions is highly disordered (Figure XIV). Similar to dislocation avalanches in plastically deformed crystals, one observes a power-law distribution of microcrack avalanches preceding the eventual rupture $(117,118,119,76,77)$. These are due to finite-size criticality (120); large avalanches in a crossover region between two regimes neither of which have bulk avalanches. At small sizes and large disorder, the bonds break in a percolation-like fashion (one at a time); at large sizes a single dominant crack is nucleated in a rare event. Our finite-size scaling analysis should be generalizable into a crossover scaling theory for the process zone near the crack tip in quasi-brittle materials (Figure XIVc). Combining these ideas with a scaling theory of plasticity (as described here) could allow for a crossover theory for the process zone for ductile fracture as well.

There are two classes of models that provide a particularly clear connection to the physics of plastically deformed crystals: the theory of depinning (described in Figure XV and XVI), and the theory of jamming (in Figure XVII). Both describe the response of a rigid system to external shear, via avalanche-like rearrangements.

Depinning transitions describe motion in the presence of dirt, as for dislocations pinned primarily on second phase precipitates. First, in fields like magnetic hysteresis and noise, depinning theories provide a comprehensive and compelling framework for understanding the experimental behavior (125) and are pursued using sophisticated functional renormalizationgroup methods $(126,127,128,129,130)$. Second, in fields like earthquakes things are more controversial; the data does not discriminate so well between different models, but there is a broad consensus that scaling approaches are important and useful (131, 132, 133); Within the scaling theories, depinning models have had notable success $(134,132,135)$, but remain 
Figure XV: Depinning and criticality

\begin{tabular}{|l|c|c|c|}
\hline System & $d$ & $d^{\prime}$ & $N$ \\
\hline \hline Planar cracks & 1 & 2 & 1 \\
\hline CDWs & 3 & 3 & 1 \\
\hline Vortices & 1 & 3 & 2 \\
\hline Droplets & 1 & 2 & 1 \\
\hline Magnetic & 2 & 3 & 1 \\
\hline Plasticity & 3 & 3 & 12 \\
\hline
\end{tabular}

(a)

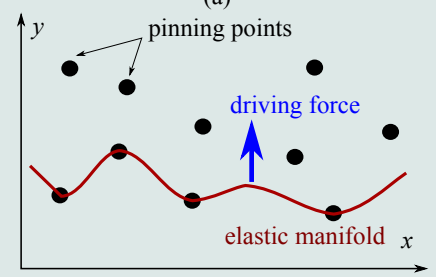

(b)

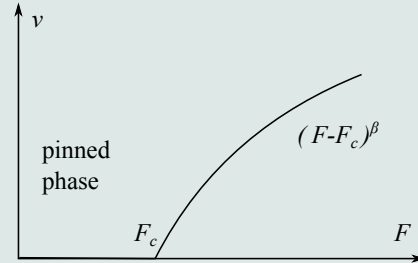

(Table) Depinning transitions describe the jerky movement of surfaces pinned by disorder as they is dragged; they have been used to describe everything from planar cracks to superconducting vortices to raindrops on windshields (121). (a) A $d$-dimensional manifold is stuck on dirt in a $d^{\prime}$-dimensional space, and can move along $N$ different directions. As the force increases, the system exhibits avalanche-like rearrangements of local regions, shifting between metastable states stuck in the dirt, until at a critical force the system starts sliding. (b) Universal power laws and scaling behavior in the velocity and velocity autocorrelation above depinning, and in the avalanche sizes, durations, shapes, and spatiotemporal correlations are explained using the emergent scale invariance at the depinning threshold. Fcc crystal plasticity is an example of a $d=d^{\prime}=3$ depinning transition, with $N=12$ slip systems yielding under an external load (although $d=d^{\prime}=2$ and $N=1$ in the simplest model $(122,123))$. Depinning transitions often have self-limiting terms that allow them to self-organize near critical points; work hardening plays this role for plasticity.

Figure XVI: Mesoscale plasticity

$$
\begin{aligned}
& \frac{1}{B} \partial_{t} \gamma(\boldsymbol{r})=\sigma_{\mathrm{ext}}+\sigma_{\mathrm{int}}(\boldsymbol{r})+\delta \sigma(\boldsymbol{r}, \gamma) \\
& \sigma_{\mathrm{int}}(\boldsymbol{k})=-\frac{G}{\pi(1-\nu) \gamma(\boldsymbol{k})} \frac{k_{x}^{2} k_{y}^{2}}{|\boldsymbol{k}|^{4}}
\end{aligned}
$$

This mesoscale plasticity evolution equation [from (122)] is typical of depinning: the evolution of the manifold $\gamma$ depends on an external driving force $\sigma_{\text {ext }}$, a nonlocal force $\sigma_{\text {int }}$ due to the rest of the manifold, and a force $\delta \sigma(\boldsymbol{r}, \gamma)$ due to dirt and other dislocations at $\boldsymbol{r}$. Here $\gamma$ is the shear strain (up to twelve components), $G$ the shear modulus, $\nu$ the Poisson ratio, and $B$ a viscoplastic rate coefficient. The nonlocal kernel given in Fourier space by the lower equation is characteristic of all elastic materials - both crystalline and amorphous (123, 124). Plasticity is different from most depinning problems for two reasons. First, the kernel is not convex (positive and negative in different directions), making most of the analytic methods challenging. Second, dislocations tangle among themselves even without dirt. Like glasses and jammed systems, they generate their own disorder as they evolve.

in competition with other types of models $(74,136,137)$. Part of the challenge is that the long-range interactions through the earths crust make the theory mean-field (138), allowing many different microscopic theories to yield the same behavior. Finally, many of the statistical theories of plasticity in crystals are depinning theories (see Figure XVI).

Jamming transitions $(139,140)$ describe the elastic response of systems near the point where the constituent particles first assemble into a rigid network. The hardness of clean 
Figure XVII: Jamming

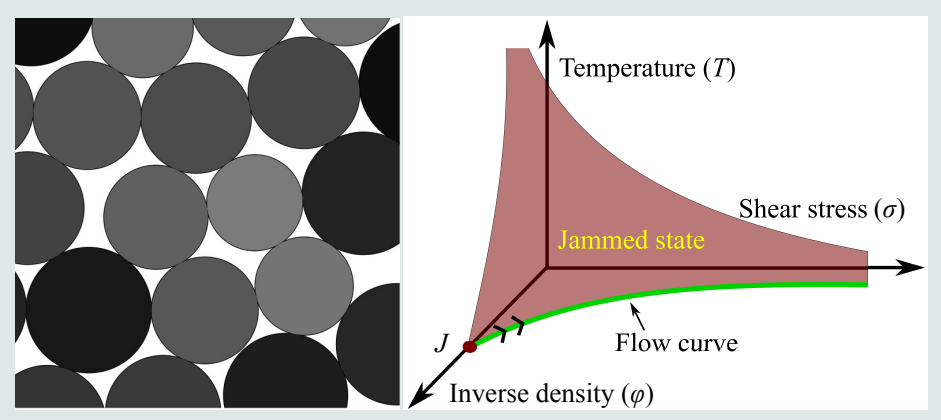

Jamming $(139,140)$ attempts to describe the onset of rigidity in molecular glasses, granular media, colloids, pastes, emulsions, foams (140), dislocation systems (141), and biological tissues (142). At low temperature, low stress, and high density the system is stuck in a jammed phase - a disordered solid state that resists shear. The jamming point $J$ controls the critical behavior of the system. In contrast to depinning, where dirt is explicitly part of the model, the disorder in a jammed system is frozen in as it jams - just as dislocations in crystals tangle themselves up as they evolve $(143,144,141)$. At point $J$ one finds the power laws, scaling collapses, and diverging length scales $(139,140)$ that are characteristic of systems with emergent scale invariance (Figure XI), although no coarse-graining approach has yet been developed. A recent scaling ansatz (145) argues that the free energy has the scaling form $F(\Delta \varphi, P, \sigma, T)=\Delta \varphi^{2} \mathcal{F}_{0}\left(P / \Delta \varphi, \sigma / \Delta \varphi^{5 / 4}, T / \Delta \varphi^{2}\right)$, where (as usual) the arguments of the scaling function are invariant under the presumed renormalization-group flow (Figure XI), with meanfield rational critical exponents in three dimensions. In particular, a point near $J$ along the zero-temperature yielding boundary (green curve in the $\varphi-\sigma$ plane), when coarse-grained, must also be a system at its yield point, farther from point $J$. This implies that the invariant combination $\sigma / \Delta \varphi^{5 / 4}$ is invariant along the yielding curve, so $\sigma_{\text {flow }} \propto \Delta \varphi^{5 / 4}(146,147,148)$. Jammed states may develop equilibrium-like properties; they seem to maximize entropy (become equally probable (20)), and to have 'thermodynamic' descriptions (see Refs. $(27,28,29,30)$ and Figure V). Perhaps the weird memory effects of Section 3 are erased by the large fluctuations near critical points.

Recrystallization: An abrupt change in the morphology of a work-hardened crystal, where the dislocations become so dense that they spontaneously annihilate, mediated by a large angle grain boundary that sweeps through the system. single crystals is primarily due to dislocation entanglement with other dislocations - the disordered pinning is not static, but dynamically evolves as the dislocations 'jam' together, making the analogy with the jamming critical point perhaps more plausible $(144,143,141)$.

In plastically deformed crystals, what is the critical point? What corresponds to the depinning force in Figure XV or point $J$ in Figure XVII? Let us consider a system where the slope of the work hardening curve $\Theta=\mathrm{d} \sigma_{Y} / \mathrm{d} \epsilon$ controls the cutoff in the avalanche size distribution, and presumably also the cutoff for the fractal self-similarity in the spatial morphology. The critical point $\Theta=0$ (which flows to self-similar fixed point under the renormalization group in Figure XI) corresponds to a material which does not work harden with increasing strain.

Such a material would either continue to deform at constant stress (perhaps related to superplasticity (149)), or the material begins to weaken with further stress (as happens in metallic glasses, leading to shear band failure $(15,16)$ ). Evidence for a 'plain old' critical point at a stress $\sigma_{\mathrm{c}}$ has been seen in micropillar experiments (73) (Figure XII(c)), where the avalanche sizes diverge at a $\sigma_{\mathrm{c}}$ that we call the critical failure stress. They find quantitative agreement with a mean-field scaling theory, for a traditional critical point, with a scaling form for the avalanche sizes $P(S \mid \sigma) \sim S^{-\tau} \mathcal{P}\left(S /\left(\sigma_{\mathrm{c}}-\sigma\right)^{-d_{f} \nu}\right)$.

Sethna et al. 
Figure XVIII: Mean field theory \& plasticity

(a)

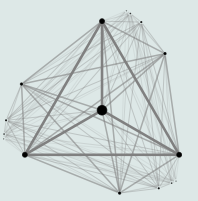

(b)

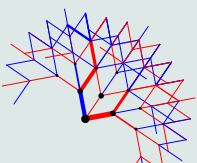

(c) Depinning $\rho(x) \sim x^{0}$ 1.0

त्र 0.8

ज़ 0.6

0.4

0.2 Yielding $\rho(x) \sim x^{\theta}$
0.0 stress threshold
Mean field theories describe systems in high dimensions or with long-range forces. Some assume each degree of freedom feels the mean of other sites like in a fully connected hypertetrahedron (a); others sit on an infinite branching tree (Bethe lattice, b). With a plastic rearrangement, the anisotropic stress increases the loading on some neighbors and decreases it on others. Some mean-field plasticity models focus on the decrease but ignore the anisotropy $(150,151)$, others argue that the anisotropy allows one to ignore the decreases $(123,152)$. (c) For systems of the former type, the density of sites close to their failure threshold must be suppressed due to past fluctuations crossing the threshold, forming a pseudogap scaling as $\left(\sigma_{\mathrm{c}}-\sigma\right)^{\theta}$. This yields a value of $\tau \leq 3 / 2$ that depends on the long-range tail in the coupling distribution. For the latter, no pseudogap appears, and the distribution of avalanche sizes scales as $P(S) \sim S^{-\tau}$ where $\tau=3 / 2$. A wide variety of experiments are consistent with $\tau=3 / 2(79,153,80,154)$. In contrast, some simulations suggest that $\tau \approx 1.3(155$, $156,157)$.

Much of the statistical physics literature describes crystal plasticity instead as a selforganized critical point (158). Depinning systems like earthquakes are thought to exhibit emergent scale invariance without fine-tuning to a critical point because the natural microscopic velocities (tectonic plates drift centimeters/year) are tiny compared to the mean velocities during avalanches (71). In many macroscopic materials, work hardening under increasing stress or strain does not peak at a critical stress or strain. ${ }^{8}$ Also, the natural scale of the work hardening coefficient $\Theta$ is comparable to the elastic modulus; one prediction (72) for the cutoff of the avalanche size distribution is a slip of $S_{0}=L^{2} E / \Theta$ - small compared to macroscopic scales, but large compared to atomic scales for large system size $L$. Thus the natural cutoff scale due to work hardening for macroscopic samples is tiny, just as the velocities of earthquake fronts are tiny, leading to a self-organized critical point. ${ }^{10}$

A key test for scaling theories of plasticity is the exponent $\tau$ giving the power law for the avalanche size distribution. Many experiments on crystalline and amorphous plasticity can be fit using a value $\tau \sim 3 / 2(152)$. While older simulations seemed to agree with this value $(160,161)$, newer simulations observe smaller values of $\tau \sim 1.25-1.4(15,155,157$,

\footnotetext{
${ }^{8}$ Instead, it terminates in a qualitative way - fracture (breaking in two), amorphization (where the crystalline correlations drop to the atomic scale), or perhaps recrystallization (159).

${ }^{9}$ We define $S$ to be the net slip. Csikor defines their avalanche size $s$ to be the strain jump, which is $b S / L^{3}$, since a slip of size $L^{2}$ causes the material to shrink by around a Burgers vector $\delta L / L \sim b / L$. Csikor's cutoff at constant load $s_{0}=b E / L \Theta$ thus becomes a cutoff in our notation of $S_{0}=L^{2} E / \Theta$.

${ }^{10}$ Here there are two different relevant variables at the fixed point, $1 / L$ and $\Theta$. The predicted scaling form for the avalanche size distribution $P(S \mid \Theta, L) \sim S^{-\tau} \mathcal{P}\left(S / L^{d_{f}}, L / \Theta^{-\nu}\right)$ gives the distribution used by Csikor et al. $P(S)=S^{-\tau} \mathrm{e}^{\left(S / S_{0}\right)^{2}}$ if we assume $\mathcal{P}(X, Y)=\exp \left(\left(X Y^{1 / \nu}\right)^{2}\right)$ and $d_{f}-1 / \nu=2$. Note that this makes sense only if $d_{f}>2$, different from that proposed by Csikor et al., but compatible with $d_{f} \sim 2.5$ found by Weiss (82).
} 
$156,162) .^{11}$

It is not clear at this time what governs these variations, but one intriguing hint is provided by two different mean-field theories for plasticity (Figure XVIII). A local rearrangement of atoms at a site will yield a net plastic slip, producing a long-range, anisotropic change in the stress. This elastic dipole adds to the imposed stress in some directions, and relieving the stress in others, often triggering disconnected pieces of the avalanche elsewhere in the system. Some mean-field theories $(152,123)$ have ignored the variation in sign of this interaction, arguing that the directional anisotropy makes the positive interactions within a slip band or glide plane the only important ones (134). This leads to a theory where every site feels a monotonically increasing external stress, which in mean-field gives the exponent $\tau=3 / 2$. Others $(165,151)$ have ignored the directional anisotropy, and argue that the random positive and negative changes in stress lead to a kind of anomalous diffusion in the distance of each site from its local yielding stress. Since any site that crosses its yielding stress will trigger an avalanche and disappear from the active list, this carves a hole out of the distribution of sites near their critical stress. This leads to fewer, larger avalanches and a lower value for $\tau$.

We end with a cautionary note, particularly addressed to the statistical physics community. Emergent scale invariance in physics leads not only to power laws and scaling functions, it also leads to universality - any two critical systems which flow to the same fixed point in Figure XI will share the same long-wavelength behavior. Thus the Ising model quantitatively describes both the disappearance of magnetism with increasing temperature in some magnets, and the disappearance of the density difference between liquid and vapor along the coexistence line as temperature and pressure are increased. In contrast, success in materials physics has historically rested upon attention to materials-specific details. Many metallurgists focus their careers on specific materials. Aluminum alloys, steels, and titanium superalloys are each worlds unto themselves. Are the scaling methods of statistical physics doomed in the attempt to describe the bewildering variety of anisotropic slip systems, dislocation mobilities, cross slip, pinning, ...?

Here we remind our statistical colleagues of a simple but profound observation by Rutenberg and Vollmayr-Lee some decades ago (Figure XIX), that our scaling theory of coarsening is an example where the 'universality class' is parameterized by entire anisotropic functions of angle. There is no good reason why this kind of strong material dependence should be incompatible with scaling and criticality. Also, there likely will be several 'universality classes' depending upon the active mechanisms for plastic deformation; systems with one active slip system behave very differently from the same material at later stages where multiple systems and cross slip become active. Our attempts to form a scaling theory must not only focus on what makes plastic deformation the same among materials composition and processing history, but also what makes each system different.

\footnotetext{
${ }^{11}$ One set of experiments (163) showed a rate-dependence yielding values of $\tau>1.5$ for slow deformations; these experiments have been modeled as a quasi-periodic approach to a critical point punctuated by quasi-periodic system-spanning events (164). 
Figure XIX: Non-universality

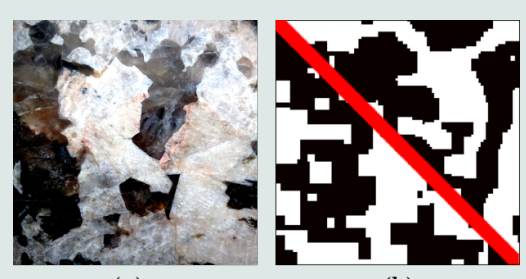

(a) Polycrystalline granite. When magma slowly cools below the Earth's surface, it coarsens into different component crystals, forming granite. Scaling theories that describe coarsening have inverse time as a relevant variable. When $r \rightarrow r / b$ times scales as $t \rightarrow t / b^{3}$ (Figure XI) (7, sec. 11.4.1). Thus salad dressing and the Ising model near $T_{c}$ are both described by the same correlation function $C(\mathbf{r}, t)=\mathcal{C}\left(r / t^{1 / 3}\right)$ since coarsening in isotropic systems is universal. In 1999, Rutenberg and Vollmayr-Lee noted that this is not true of anisotropic crystalline systems, or even the Ising model away from $T_{c}$; although a scaling form exists, it is dependent on the anisotropic surface tensions and interface mobilities. (166) Panel (b) shows a comparison of coarsening in the 3D Ising model (upper triangle) to the same system with additional weak antiferromagnetic next-neighbor bonds (lower triangle). The added interaction slows coarsening to $r \sim \log (t)$ and introduces a preference for flat boundaries aligned with the lattice directions (167), producing facets like those in granite (a). Although physicists are fond of universality, the world is filled with a bewildering variety of rocks and dislocation tangles. Many materials-dependent properties likely must be treated as "relevant variables" in our eventual scaling theory of plasticity, as they are for coarsening.

\section{Conclusions}

\section{SUMMARY POINTS}

1. Crystals are a challenge for nonequilibrium statistical mechanics. Our methods addressing disorder, long-range forces, constrained dynamics, and anisotropic interactions must be combined to address dislocation entanglement in crystal plasticity.

2. There are many rigid statistical mechanical systems with behavior closely analogous to the yield stress and work hardening seen in crystal plasticity. Upon unloading, these simpler systems are left in configurations that are rare among the possible metastable states, encoding the material history in interesting ways.

3. The thermal and mechanical history of a crystal thus is probably encoded in its morphology. This need not prevent a phenomenological theory from effectively encapsulating its future macroscale behavior.

4. Avalanche dynamics and the morphologies of dislocation tangles provide clear evidence that plastically deformed crystals exhibit an emergent scale invariance.

5. Strong analogies can be made between plasticity avalanches and scaling behavior seen in fracture, depinning, jamming, and even dilute colloidal suspensions under shear.

6. Crystal plasticity is immensely complex and strongly material and history dependent. A successful renormalization-group scaling theory of plasticity in crystals will depend on far more details about the microscopic behavior than has been typical in previous systems exhibiting scale invariance. 


\section{DISCLOSURE STATEMENT}

The authors are not aware of any affiliations, memberships, funding, or financial holdings that might be perceived as affecting the objectivity of this review.

\section{ACKNOWLEDGMENTS}

We thank Bulbul Chakraborty, Karen Daniels, Andrea J. Liu, and M. Lisa Manning for extensive consultation. We also thank Paul Dawson, Ryan Elliott, Susan Coppersmith, James Jenkins, and Ellad Tadmor for kindly providing references and/or permission to reprint figures. JPS, MKB DBL and AR were supported by the Department of Energy through Grant No. DE-FG02-07ER46393. LXH, JPK, EDL and KNQ were supported by the National Science Foundation through Grant No. NSF DMR-1312160. KD is thankful for the support from NSF through Grant No. NSF CBET 1336634, and also thanks the Kavli Institute of theoretical Physics for hospitality and support through Grant No. NSF PHY11-25915. CPG is supported by the National Science Foundation through the Harvard Materials Research Science and Engineering Center DMR1420570, and the Division of Mathematical Sciences DMS-1411694. JRG and XN are grateful to the U.S. Department of Energy (DOE) through J.R.G.s Early Career Research Program under grant DE-SC0006599. LXH was supported by a fellowship from Cornell University. EDL thanks support by the National Science Foundation through the fellowship No. NSF GRFP (DGE-1650441). KNQ was supported by the Natural Sciences and Engineering Research Council of Canada. DZR thanks support by the Bethe/KIC Fellowship, and the National Science Foundation Grant No. NSF DMR1308089. AS acknowledges support from the Miller Fellowship by the Miller Institute for Basic Research in Science, at UC Berkeley. SZ is supported by the European Research Council advanced grant SIZEFFECTS.

\section{LITERATURE CITED}

1. Chandler D. 1987. Introduction to modern statistical mechanics. Oxford University Press

2. Forster D. 1995. Hydrodynamic fluctuations, broken symmetry, and correlation functions. Advanced book classics. Perseus Books

3. Martin PC. 1968. Problème á n corps (many body physics). Gordon and Breach, New York, $37-136$

4. Landau L, Lifshitz E. 2013. Statistical physics. No. v. 5. Elsevier Science

5. Tadmor EB, Miller RE, Elliott RS. 2012. Continuum mechanics and thermodynamics: from fundamental concepts to governing equations. Cambridge University Press

6. Anderson PW. 1984. Lectures on amorphous systems

7. Sethna JP. 2006. Statistical mechanics: Entropy, order parameters, and complexity, http: //www.physics.cornell.edu/sethna/StatMech/. Oxford: Oxford University Press

8. Chui ST, Weeks JD. 1978. Dynamics of the roughening transition. Phys. Rev. Lett. 40:733-736

9. Liarte DB, Bierbaum M, Mosna RA, Kamien RD, Sethna JP. 2016. Weirdest martensite: Smectic liquid crystal microstructure and Weyl-Poincaré invariance. Phys. Rev. Lett. 116:147802

10. Vegge T, Sethna JP, Cheong SA, Jacobsen KW, Myers CR, Ralph DC. 2001. Calculation of quantum tunneling for a spatially extended defect: The dislocation kink in copper has a low effective mass. Physical Review Letters 86:1546-1549

11. Hull D, Bacon DJ. 2011. Introduction to dislocations, vol. 37. Elsevier

12. Ni X, Greer JR. 2016. Unpublished

13. Bulatov VV, Hsiung LL, Tang M, Arsenlis A, Bartelt MC, et al. 2006. Dislocation multijunctions and strain hardening. Nature 440:1174-1178 
14. Taylor GI. 1934. The mechanism of plastic deformation of crystals. part i. theoretical. Proceedings of the Royal Society of London A: Mathematical, Physical and Engineering Sciences 145:362-387

15. Budrikis Z, Zapperi S. 2013. Avalanche localization and crossover scaling in amorphous plasticity. Phys. Rev. E 88:062403

16. Sandfeld S, Budrikis Z, Zapperi S, Castellanos DF. 2015. Avalanches, loading and finite size effects in 2d amorphous plasticity: results from a finite element model. Journal of Statistical Mechanics: Theory and Experiment 2015:P02011

17. Dieter GE, Bacon DJ. 1986. Mechanical metallurgy, vol. 3. McGraw-Hill New York

18. Bi D, Henkes S, Daniels KE, Chakraborty B. 2015. The statistical physics of athermal materials. Annual Review of Condensed Matter Physics 6:63-83

19. Edwards S, Oakeshott R. 1989. Theory of powders. Physica A: Statistical Mechanics and its Applications 157:1080 - 1090

20. Martiniani S, Schrenk KJ, Ramola K, Chakraborty B, Frenkel D. 2016. Are some packings more equal than others? A direct test of the Edwards conjecture. arXiv:1610.06328

21. Henkes S, Chakraborty B. 2005. Jamming as a critical phenomenon: A field theory of zerotemperature grain packings. Phys. Rev. Lett. 95:198002

22. Edwards S. 2005. The full canonical ensemble of a granular system. Physica A: Statistical Mechanics and its Applications 353:114-118

23. Blumenfeld R, Edwards S. 2009. On granular stress statistics: Compactivity, angoricity, and some open issues. JOURNAL OF PHYSICAL CHEMISTRY B 113:3981-3987

24. Jenkins JT. 2016. Kinetic theories for collisional grain flows. Handbook of Granular Materials :155

25. Puckett JG, Daniels KE. 2013. Equilibrating temperaturelike variables in jammed granular subsystems. Phys. Rev. Lett. 110:058001

26. Langer J, Bouchbinder E, Lookman T. 2010. Thermodynamic theory of dislocation-mediated plasticity. Acta Materialia 58:3718-3732

27. Makse HA, Kurchan J. 2002. Testing the thermodynamic approach to granular matter with a numerical model of a decisive experiment. Nature 415:614-617

28. Abate AR, Durian DJ. 2008. Effective temperatures and activated dynamics for a twodimensional air-driven granular system on two approaches to jamming. Phys. Rev. Lett. 101:245701

29. Berthier L, Barrat JL. 2002. Shearing a glassy material: numerical tests of nonequilibrium mode-coupling approaches and experimental proposals. Physical review letters 89:095702

30. Ono IK, O'Hern CS, Durian DJ, Langer SA, Liu AJ, Nagel SR. 2002. Effective temperatures of a driven system near jamming. Phys. Rev. Lett. 89:095703

31. Jonason K, Vincent E, Hammann J, Bouchaud J, Nordblad P. 1998. Memory and chaos effects in spin glasses. Physical Review Letters 81:3243

32. Sethna JP, Dahmen K, Kartha S, Krumhansl JA, Roberts BW, Shore JD. 1993. Hysteresis and hierarchies - dynamics of disorder-driven 1st-order phase-transformations. Physical Review Letters 70:3347-3350

33. Coppersmith S. 1987. A simple illustration of phase organization. Physics Letters A 125:473475

34. Coppersmith S, Littlewood P. 1987. Pulse-duration memory effect and deformable chargedensity waves. Physical Review B 36:311

35. Tang C, Wiesenfeld K, Bak P, Coppersmith S, Littlewood P. 1987. Phase organization. Physical review letters $58: 1161$

36. Asaro R, Lubarda V. 2006. Mechanics of solids and materials. Cambridge books online. Cambridge University Press

37. de Silva C. 2013. Mechanics of materials. Computational Mechanics and Applied Analysis. CRC Press 
38. Nair S. 2015. Mechanics of aero-structures. Cambridge University Press

39. Philpot T. 2012. Mechanics of materials: An integrated learning system, 3rd edition: Third edition. Wiley Global Education

40. Machta BB, Chachra R, Transtrum M, Sethna JP. 2013. Parameter space compression underlies emergent theories and predictive models. Science 342:604-607

41. Transtrum MK, Machta BB, Brown KS, Daniels BC, Myers CR, Sethna JP. 2015. Perspective: Sloppiness and emergent theories in physics, biology, and beyond. The Journal of Chemical Physics 143:-

42. Kocks UF, Tomé CN, Wenk HR. 2000. Texture and anisotropy: preferred orientations in polycrystals and their effect on materials properties. Cambridge university press

43. Randle V, Engler O. 2000. Texture analysis. Macrotexture, Microtexture and Orientation Mapping, Gordon and Breach, London

44. Bhattacharya K. 2003. Microstructure of martensite: Why it forms and how it gives rise to the shape-memory effect. Oxford University Press, Oxford

45. Corté L, Chaikin PM, Gollub JP, Pine DJ. 2008. Random organization in periodically driven systems. Nature Physics 4:420 - 424

46. Pine D, Gollub J, Brady J, Leshansky A. 2005. Chaos and threshold for irreversibility in sheared suspensions. Nature 438:9971000

47. Paulsen JD, Keim NC, Nagel SR. 2014. Multiple transient memories in experiments on sheared non-brownian suspensions. Phys. Rev. Lett. 113

48. Reichhardt C, Reichhardt CJO. 2009. Random organization and plastic depinning. Phys. Rev. Lett. 103

49. Keim NC, Arratia PE. 2013. Yielding and microstructure in a 2 d jammed material under shear deformation. Soft Matter 9:6222-6225

50. Keim NC, Arratia PE. 2014. Mechanical and microscopic properties of the reversible plastic regime in a 2d jammed material. Phys. Rev. Lett. 112

51. Menon GI, Ramaswamy S. 2009. Universality class of the reversible-irreversible transition in sheared suspensions. Phys. Rev. E 79

52. Regev I, Weber J, Reichhardt C, Dahmen KA, Lookman T. 2015. Reversibility and criticality in amorphous solids. Nature Communications 6

53. Regev I, Lookman T, Reichhardt C. 2013. Onset of irreversibility and chaos in amorphous solids under periodic shear. Phys. Rev. E 88

54. Fiocco D, Foffi G, Sastry S. 2013. Oscillatory athermal quasistatic deformation of a model glass. Phys. Rev. E 88

55. Jeanneret R, Bartolo D. 2014. Geometrically protected reversibility in hydrodynamic loschmidt-echo experiments. Nat. Commun. 5

56. Nagamanasa KH, Gokhale S, Sood AK, Ganapathy R. 2014. Experimental signatures of a nonequilibrium phase transition governing the yielding of a soft glass. Phys. Rev. E Stat. Nonlin. Soft Matter Phys. 89

57. Rogers MC, Chen K, Andrzejewski L, Narayanan S, Ramakrishnan S, et al. 2014. Echoes in $\mathrm{x}$-ray speckles track nanometer-scale plastic events in colloidal gels under shear. Phys. Rev. E Stat. Nonlin. Soft Matter Phys. 90

58. Möbius R, Heussinger C. 2014. (ir)reversibility in dense granular systems driven by oscillating forces. Soft Matter 10:48064812

59. Schreck CF, Hoy RS, Shattuck MD, OHern CS. 2013. Particle-scale reversibility in athermal particulate media below jamming. Phys. Rev. E Stat. Nonlin. Soft Matter Phys. 88

60. Slotterback Sea. 2012. Onset of irreversibility in cyclic shear of granular packings. Phys. Rev. E Stat. Nonlin. Soft Matter Phys. 85

61. Royer JR, Chaikin PM. 2015. Precisely cyclic sand: self-organization of periodically sheared frictional grains. Proc. Natl Acad. Sci. 112:4953

62. Zhou C, Olson Reichhardt C, Reichhardt C, Beyerlein I. 2014. Random organization in peri- 
odically driven gliding dislocations. Phys. Lett. A 378

63. Okuma S, Tsugawa Y, Motohashi A. 2011. Transition from reversible to irreversible flow: Absorbing and depinning transitions in a sheared-vortex system. Phys. Rev. B 83

64. Mangan N, Reichhardt C, Reichhardt C. 2008. Reversible to irreversible flow transition in periodically driven vortices. Phys. Rev. Lett. 100

65. Pérez Daroca D, Pasquini G, Lozano GS, Bekeris V. 2011. Dynamics of superconducting vortices driven by oscillatory forces in the plastic-flow regime. Phys. Rev. B 84

66. López D, Kwok WK, Safar H, Olsson RJ, Petrean AM, et al. 1999. Spatially resolved dynamic correlation in the vortex state of high temperature superconductors. Phys. Rev. Lett. 82:12771280

67. Miguel MC, Zapperi S. 2003. Tearing transition and plastic flow in superconducting thin films. Nature Mat. 2

68. Shaw G, Mandal P, Banerjee SS, Niazi A, Rastogi AK, et al. 2012. Critical behavior at depinning of driven disordered vortex matter in 2h-nbs2. Phys. Rev. B 85

69. Okuma S, Motohashi A. 2012. Critical behavior associated with transient dynamics near the depinning transition. New J. Phys. 14

70. Goldenfeld N. 1992. Lectures on phase transitions and the renormalization group

71. Sethna JP, Dahmen KA, Myers CR. 2001. Crackling noise. Nature 410:242-250

72. Csikor FF, Motz C, Weygand D, Zaiser M, Zapperi S. 2007. Dislocation avalanches, strain bursts, and the problem of plastic forming at the micrometer scale. Science 318:251-254

73. Friedman N, Jennings AT, Tsekenis G, Kim JY, Tao M, et al. 2012. Statistics of dislocation slip avalanches in nanosized single crystals show tuned critical behavior predicted by a simple mean field model. Phys. Rev. Lett. 109:095507

74. Burridge R, Knopoff L. 1967. Model and theoretical seismicity. Bulletin of the seismological society of america $57: 341-371$

75. Bak P, Tang C. 1989. Earthquakes as a self-organized critical phenomenon. J. geophys. Res 94:635-15

76. Petri A, Paparo G, Vespignani A, Alippi A, Costantini M. 1994. Experimental evidence for critical dynamics in microfracturing processes. Physical review letters 73:3423

77. Garcimartin A, Guarino A, Bellon L, Ciliberto S. 1997. Statistical properties of fracture precursors. Physical Review Letters 79:3202

78. Chen Y, Papanikolaou S, Sethna JP, Zapperi S, Durin G. 2011. Avalanche spatial structure and multivariable scaling functions; sizes, heights, widths, and views through windows. Physical Review E 84:061103

79. Papanikolaou S, Bohn F, Sommer RL, Durin G, Zapperi S, Sethna JP. 2011. Universality beyond power laws and the average avalanche shape. Nature Physics 7:316-320

80. Miguel MC, Vespignani A, Zapperi S, Weiss J, Grasso JR. 2001. Intermittent dislocation flow in viscoplastic deformation. Nature 410:667-671

81. Dimiduk DM, Woodward C, LeSar R, Uchic MD. 2006. Scale-free intermittent flow in crystal plasticity. Science 312:1188-1190

82. Weiss J, Marsan D. 2003. Three-dimensional mapping of dislocation avalanches: Clustering and space/time coupling. Science 299

83. Mughrabi H. 1983. Dislocation wall and cell structures and long-range internal stresses in deformed metal crystals. Acta metallurgica 31:1367-1379

84. Hähner P, Bay K, Zaiser M. 1998. Fractal dislocation patterning during plastic deformation. Physical review letters 81:2470

85. Hughes D, Chrzan D, Liu Q, Hansen N. 1998. Scaling of misorientation angle distributions. Physical review letters 81:4664

86. Chen YS, Choi W, Papanikolaou S, Bierbaum M, Sethna JP. 2013. Scaling theory of continuum dislocation dynamics in three dimensions: Self-organized fractal pattern formation. International Journal of Plasticity 46:94-129 
87. Chen YS, Choi W, Papanikolaou S, Sethna JP. 2010. Bending crystals: the evolution of grain boundaries and fractal dislocation structures. Phys. Rev. Lett. 105:105501

88. Bertalan Z, Shekhawat A, Sethna JP, Zapperi S. 2014. Fracture strength: Stress concentration, extreme value statistics, and the fate of the weibull distribution. Phys. Rev. Applied 2:034008

89. Kent-Dobias J, Shekhawat A, Sethna JP. 2016. (work in progress)

90. Alava MJ, Nukala PK, Zapperi S. 2006. Morphology of two-dimensional fracture surfaces. Journal of Statistical Mechanics: Theory and Experiment 2006:L10002

91. Zapperi S, Nukala PKV, Šimunović S. 2005. Crack roughness and avalanche precursors in the random fuse model. Physical Review E 71:026106

92. Alava MJ, Nukala PK, Zapperi S. 2006. Statistical models of fracture. Advances in Physics $55: 349-476$

93. Bouchaud E, Lapasset G, Planes J. 1990. Fractal dimension of fractured surfaces: a universal value? EPL (Europhysics Letters) 13:73

94. Bouchaud E. 1997. Scaling properties of cracks. Journal of Physics: Condensed Matter 9:4319

95. Ponson L, Bonamy D, Bouchaud E. 2006. Two-dimensional scaling properties of experimental fracture surfaces. Physical review letters 96:035506

96. Morel S, Schmittbuhl J, Bouchaud E, Valentin G. 2000. Scaling of crack surfaces and implications for fracture mechanics. Physical review letters 85:1678

97. Måløy KJ, Hansen A, Hinrichsen EL, Roux S. 1992. Experimental measurements of the roughness of brittle cracks. Physical Review Letters 68:213

98. Hansen A, Schmittbuhl J. 2003. Origin of the universal roughness exponent of brittle fracture surfaces: stress-weighted percolation in the damage zone. Physical review letters 90:045504

99. Schmittbuhl J, Hansen A, Batrouni GG. 2003. Roughness of interfacial crack fronts: stressweighted percolation in the damage zone. Physical review letters 90:045505

100. Laurson L, Santucci S, Zapperi S. 2010. Avalanches and clusters in planar crack front propagation. Physical Review E 81:046116

101. Schmittbuhl J, Roux S, Vilotte JP, Måløy KJ. 1995. Interfacial crack pinning: effect of nonlocal interactions. Physical Review Letters 74:1787

102. Rosso A, Krauth W. 2002. Roughness at the depinning threshold for a long-range elastic string. Physical Review E 65:025101

103. Mecholsky J, Passoja D, Feinberg-Ringel K. 1989. Quantitative analysis of brittle fracture surfaces using fractal geometry. Journal of the American Ceramic Society 72:60-65

104. Mecholsky J, Mackin T, Passoja D. 1988. Self-similar crack propagation in brittle materials. Fractography of Glasses and Ceramics Westerville, Ohio, 1988, 22:127-134

105. Mecholsky JJ, Freiman SW. 1991. Relationship between fractal geometry and fractography. Journal of the American Ceramic Society 74:3136-3138

106. Tsai Y, Mecholsky J. 1991. Fractal fracture of single crystal silicon. Journal of Materials Research 6:1248-1263

107. Schmittbuhl J, Måløy KJ. 1997. Direct observation of a self-affine crack propagation. Physical review letters $78: 3888$

108. Schmittbuhl J, Schmitt F, Scholz C. 1995. Scaling invariance of crack surfaces. Journal of Geophysical Research: Solid Earth 100:5953-5973

109. Schmittbuhl J, Gentier S, Roux S. 1993. Field measurements of the roughness of fault surfaces. Geophysical Research Letters 20:639-641

110. Santucci S, Måløy KJ, Delaplace A, Mathiesen J, Hansen A, et al. 2007. Statistics of fracture surfaces. Physical review E 75:016104

111. Chen YJ, Zapperi S, Sethna JP. 2015. Crossover behavior in interface depinning. Phys. Rev. E 92:022146

112. Talreja R, Weibull W. 2013. Probability of fatigue failure based on residual strength. In ICF4, Waterloo (Canada) 1977

113. Jayatilaka AdS, Trustrum K. 1977. Statistical approach to brittle fracture. Journal of Mate- 
rials Science 12:1426-1430

114. Phoenix SL, Taylor HM. 1973. The asymptotic strength distribution of a general fiber bundle. Advances in Applied Probability :200-216

115. Györgyi G, Moloney N, Ozogány K, Rácz Z, Droz M. 2010. Renormalization-group theory for finite-size scaling in extreme statistics. Physical Review E 81:041135

116. Györgyi G, Moloney N, Ozogány K, Rácz Z. 2008. Finite-size scaling in extreme statistics. Physical review letters 100:210601

117. Salminen L, Tolvanen A, Alava MJ. 2002. Acoustic emission from paper fracture. Physical Review Letters 89:185503

118. Koivisto J, Rosti J, Alava MJ. 2007. Creep of a fracture line in paper peeling. Physical review letters 99:145504

119. Hemmer PC, Hansen A. 1992. The distribution of simultaneous fiber failures in fiber bundles. Journal of applied mechanics 59:909-914

120. Shekhawat A, Zapperi S, Sethna JP. 2013. From damage percolation to crack nucleation through finite-size criticality. Physical Review Letters 110:185505

121. Fisher DS. 1998. Collective transport in random media: from superconductors to earthquakes. Physics Reports 301:113 - 150

122. Zaiser M, Moretti P. 2005. Fluctuation phenomena in crystal plasticitya continuum model. Journal of Statistical Mechanics: Theory and Experiment 2005:P08004

123. Zaiser M. 2006. Scale invariance in plastic flow of crystalline solids. Advances in Physics $55: 185-245$

124. Talamali M, Petäjä V, Vandembroucq D, Roux S. 2011. Avalanches, precursors, and finite-size fluctuations in a mesoscopic model of amorphous plasticity. Phys. Rev. E 84:016115

125. Durin G, Zapperi S. 2006. Chapter 3 - the barkhausen effect. In The Science of Hysteresis, eds. G Bertotti, ID Mayergoyz. Oxford: Academic Press, $181-267$

126. Narayan O, Fisher DS. 1993. Threshold critical dynamics of driven interfaces in random media. Phys. Rev. B 48:7030-7042

127. Leschhorn H, Nattermann T, Stepanow S, Tang LH. 1997. Driven interface depinning in a disordered medium. Annalen der Physik 509:1-34

128. Chauve P, Giamarchi T, Le Doussal P. 2000. Creep and depinning in disordered media. Phys. Rev. B 62:6241-6267

129. Chauve P, Le Doussal P, Jörg Wiese K. 2001. Renormalization of pinned elastic systems: How does it work beyond one loop? Phys. Rev. Lett. 86:1785-1788

130. Le Doussal P, Wiese KJ, Chauve P. 2002. Two-loop functional renormalization group theory of the depinning transition. Phys. Rev. B 66:174201

131. Kagan YY. 2010. Earthquake size distribution: Power-law with exponent $\beta=1 / 2$ ? Tectonophysics 490:103 - 114

132. Ben-Zion Y. 2008. Collective behavior of earthquakes and faults: Continuum-discrete transitions, progressive evolutionary changes, and different dynamic regimes. Reviews of Geophysics 46:n/a-n/a. RG4006

133. Baró J, Corral A, Illa X, Planes A, Salje EKH, et al. 2013. Statistical similarity between the compression of a porous material and earthquakes. Phys. Rev. Lett. 110:088702

134. Fisher DS, Dahmen KA, Ramanathan D, Ben-Zion Y. 1997. Statistics of earthquakes in simple models of heterogeneous faults. Phys. Rev. Lett. 78:4885-4888

135. Chen K, Bak P, Obukhov SP. 1991. Self-organized criticality in a crack-propagation model of earthquakes. Phys. Rev. A 43:625-630

136. Carlson JM, Langer JS. 1989. Properties of earthquakes generated by fault dynamics. Phys. Rev. Lett. 62:2632-2635

137. Langer J, Carlson J, Myers CR, Shaw BE. 1996. Slip complexity in dynamic models of earthquake faults. Proceedings of the National Academy of Sciences 93:3825-3829

138. Dahmen K, Ertaş D, Ben-Zion Y. 1998. Gutenberg-richter and characteristic earthquake be- 
havior in simple mean-field models of heterogeneous faults. Phys. Rev. E 58:1494-1501

139. Liu AJ, Nagel SR. 2010. The jamming transition and the marginally jammed solid. Annual Review of Condensed Matter Physics 1:347-369

140. Liu AJ, Nagel SR, van Saarloos W, Wyart M. 2011. The jamming scenario-an introduction and outlook. Oxford Scholarship Online: September 2011, 1-72

141. Ispánovity PD, Laurson L, Zaiser M, Groma I, Zapperi S, Alava MJ. 2014. Avalanches in 2d dislocation systems: Plastic yielding is not depinning. Phys. Rev. Lett. 112:235501

142. Bi D, Yang X, Marchetti MC, Manning ML. 2016. Motility-driven glass and jamming transitions in biological tissues. Phys. Rev. X 6:021011

143. Tsekenis G, Goldenfeld N, Dahmen KA. 2011. Dislocations jam at any density. Phys. Rev. Lett. 106:105501

144. Miguel MC, Vespignani A, Zaiser M, Zapperi S. 2002. Dislocation jamming and andrade creep. Phys. Rev. Lett. 89:165501

145. Goodrich CP, Liu AJ, Sethna JP. 2016. Scaling ansatz for the jamming transition. Proceedings of the National Academy of Sciences 113

146. Hatano T. 2008. Scaling properties of granular rheology near the jamming transition. Journal of the Physical Society of Japan 77:123002

147. Tighe BP, Woldhuis E, Remmers JJC, van Saarloos W, van Hecke M. 2010. Model for the scaling of stresses and fluctuations in flows near jamming. Phys. Rev. Lett. 105:088303

148. Dinkgreve M, Paredes J, Michels MAJ, Bonn D. 2015. Universal rescaling of flow curves for yield-stress fluids close to jamming. Phys. Rev. E 92:012305

149. Nieh TG, Wadsworth J, Sherby OD. 2005. Superplasticity in metals and ceramics. Cambridge university press

150. Pázmándi F, Zaránd G, Zimányi GT. 2000. Self-organized criticality in the hysteresis of the Sherrington-Kirkpatrick model. Physica B: Condensed Matter 275:207-211

151. Lin J, Wyart M. 2016. Mean-field description of plastic flow in amorphous solids. Phys. Rev. $X$ 6:011005

152. Dahmen KA, Ben-Zion Y, Uhl JT. 2009. Micromechanical Model for Deformation in Solids with Universal Predictions for Stress-Strain Curves and Slip Avalanches. Physical Review Letters 102:175501

153. Sun BA, Yu HB, Jiao W, Bai HY, Zhao DQ, Wang WH. 2010. Plasticity of Ductile Metallic Glasses: A Self-Organized Critical State. Physical Review Letters 105:035501

154. Antonaglia J, Wright WJ, Gu X, Byer RR, Hufnagel TC, et al. 2014. Bulk Metallic Glasses Deform via Slip Avalanches. Physical Review Letters 112:155501

155. Liu C, Ferrero EE, Puosi F, Barrat JL, Martens K. 2016. Driving Rate Dependence of Avalanche Statistics and Shapes at the Yielding Transition. Physical Review Letters 116:065501

156. Budrikis Z, Fernandez-Castellanos D, Sandfeld S, Zaiser M, Zapperi S. 2015. Universality of Avalanche Exponents in Plastic Deformation of Disordered Solids

157. Salerno KM, Robbins MO. 2013. Effect of inertia on sheared disordered solids: Critical scaling of avalanches in two and three dimensions. Phys. Rev. E 88:062206-15

158. Bak P, Tang C, Wiesenfeld K. 1988. Self-organized criticality. Phys. Rev. A 38:364-374

159. Rollett A, Humphreys F, Rohrer GS, Hatherly M. 2004. Recrystallization and related annealing phenomena. Elsevier

160. Csikor FF, Motz C, Weygand D, Zaiser M, Zapperi S. 2007. Dislocation avalanches, strain bursts, and the problem of plastic forming at the micrometer scale. Science 318:251-254

161. Zaiser M, Marmo B, Moretti P. 2005. The yielding transition in crystal plasticity-discrete dislocations and continuum models. In International Conference on Statistical Mechanics of Plasticity and Related Instabilities, Indian Institute of Science, Bangalore, India

162. Lehtinen A, Costantini G, Alava MJ, Zapperi S, Laurson L. 2016. Glassy features of crystal plasticity. Phys. Rev. B 94:064101 
163. Papanikolaou S, Dimiduk DM, Choi W, Sethna JP, Uchic MD, et al. 2012. Quasi-periodic events in crystal plasticity and the self-organized avalanche oscillator. Nature 490:517-521

164. Jagla EA, Landes FmcP, Rosso A. 2014. Viscoelastic effects in avalanche dynamics: A key to earthquake statistics. Phys. Rev. Lett. 112:174301

165. Pázmándi F, Zaránd G, Zimányi GT. 1999. Self-organized criticality in the hysteresis of the sherrington-kirkpatrick model. Phys. Rev. Lett. 83:1034-1037

166. Rutenberg AD, Vollmayr-Lee BP. 1999. Anisotropic coarsening: Grain shapes and nonuniversal persistence. Phys. Rev. Lett. 83:3772-3775

167. Shore JD, Holzer M, Sethna JP. 1992. Logarithmically slow domain growth in nonrandomly frustrated systems - Ising-models with competing interactions. Physical Review B 46:1137611404 